\title{
Observer-Based Robust Adaptive Fuzzy Control for MIMO Nonlinear Uncertain Systems with Delayed Output
}

\author{
Chiang Cheng Chiang \\ Department of Electrical Engineering, Tatung University, 40 Chung-Shan North Road, Sec. 3, Taipei, Taiwan \\ Correspondence should be addressed to Chiang Cheng Chiang; ccchiang@ttu.edu.tw
}

Received 29 October 2012; Revised 20 January 2013; Accepted 22 January 2013

Academic Editor: Peng Shi

Copyright ( $) 2013$ Chiang Cheng Chiang. This is an open access article distributed under the Creative Commons Attribution License, which permits unrestricted use, distribution, and reproduction in any medium, provided the original work is properly cited.

\begin{abstract}
An observer-based robust adaptive fuzzy control scheme is presented to tackle the problem of the robust stability and the tracking control for a class of multiinput multioutput (MIMO) nonlinear uncertain systems with delayed output. Because the nonlinear system functions and the uncertainties of the controlled system including structural uncertainties are supposed to be unknown, fuzzy logic systems are utilized to approximate these nonlinear system functions and the upper bounded functions of the uncertainties. Moreover, the upper bound of uncertainties caused by these fuzzy modeling errors is also estimated. In addition, the state observer based on state variable filters is designed to estimate all states which are not available for measurement in the controlled system. By constructing an appropriate Lyapunov function and using strictly positive-real (SPR) stability theorem, the proposed robust adaptive fuzzy controller not only guarantees the robust stability of a class of multivariable nonlinear uncertain systems with delayed output but also maintains a good tracking performance. Finally, some simulation results are illustrated to verify the effectiveness of the proposed control approach.
\end{abstract}

\section{Introduction}

Controller design for nonlinear systems has been given a lot of attention in the control community during the last two decades. Recently, the methods of feedback linearization have been successfully applied in the development of controllers for a class of nonlinear systems [1,2]. However, the above techniques can be only applied to nonlinear systems whose dynamics are exactly known. Actually, it is difficult to obtain the exact construction of the systems in most of industrial and engineering systems because of the complexity of systems. In addition, the existence of uncertainties such as parameter uncertainties, modeling errors, and external disturbances may lead to poor performance and instability [3-8]. Hence, the study of robust stability of nonlinear uncertain systems in the presence of uncertainties is an important topic for the control design engineer.

Recently, fuzzy control technique has been considered extensively in the control problems of complex and ill-defined nonlinear systems in the presence of incomplete knowledge of the plant [3,9-15]. However, the above adaptive fuzzy controllers are only limited to the systems under the conservative assumption that system states are available for measurement. In order to relax this restriction, an observer-based fuzzy adaptive output feedback control for the SISO nonlinear systems is presented to tackle the nonlinear systems whose states are not available [16-23]. So far, few research results have been extended to MIMO nonlinear systems [24, 25]. The common feature of most previous results $[16,19,24$, $25]$ is the assumption that the controlled systems are free of uncertainties. Although the above restrictive assumption can be relaxed in $[17,18,20-23]$, the uncertainties are assumed to be a bounded external disturbance. Therefore, the motivation of this paper is to synthesize an observer-based robust adaptive fuzzy control scheme to deal with the tracking control problem for a class of MIMO nonlinear uncertain systems with delayed output in the presence of uncertainties including the structural uncertainty.

On the other hand, the design problem of nonlinear timedelay systems has received considerable attention in [2631] because time-delay characteristic often encountered in 
various engineering systems may not only cause instability but also lead to serious deterioration in the performance of the plants. By employing the input-output approach and the scaled small gain theorem, the filtering problem for discretetime T-S fuzzy systems with time-varying delay has been studied [26]. The robust $H_{\infty}$ control problem for stochastic systems with a delay in the state is investigated in [28], and the results are further extended to the stochastic timedelay systems with parameter uncertainties. The problems of stability analysis and robust control for uncertain systems with input delay were examined in [29, 31]. In [27], the design scheme of output feedback controller for a class of SISO nonlinear systems with delayed output was proposed to construct a delay-dependent controller making the closed-loop system globally asymptotically stable. In [30], a new approach for the construction of a state observer for SISO nonlinear systems with delayed output was presented to ensure the global exponential convergence to zero of the observation error. It should be pointed out that the uncertainty was not taken into account in $[27,30]$. Therefore, the robust control problem of MIMO nonlinear systems with delayed output in the presence of uncertainties will be considered in this paper.

In this paper, the problem of controller design for a class of MIMO nonlinear uncertain output-delay systems whose states are not available is considered. The main features of the proposed observer-based robust adaptive fuzzy controller are summarized as follows. (i) Fuzzy logic systems with some appropriate learning laws are applied to approximate the nonlinear system functions and the upper bounded functions of the uncertainties including the structural uncertainty. (ii) The unknown upper bound of the uncertainties caused by approximation (or fuzzy modeling) error is estimated by a simple adaptive law. (iii) The state observer based on state variable filters is designed to estimate all states which are not available for measurement in the controlled system. (iv) By constructing an appropriate Lyapunov function and using strictly positive-real (SPR) stability theorem, the proposed robust adaptive fuzzy output feedback controller can not only guarantee the robust stability of the whole closed-loop system but also obtain the good tracking performance.

This paper is organized as follows: the description of the system and the concept of fuzzy logic systems are given together in Section 2. Section 3 proposes an observer-based robust adaptive fuzzy output feedback controller to achieve the purpose of asymptotic stabilization and output tracking performance of the whole closed-loop MIMO nonlinear uncertain systems with delayed output. In Section 4, a series of simulation results are illustrated to show the effectiveness of the proposed control scheme. Finally, a conclusion is given in Section 5.

\section{Problem Formulation and Preliminaries}

2.1. Problem Formulation. Consider a class of MIMO nonlinear uncertain systems with delayed output in the following form:

$$
\begin{aligned}
& \dot{x}_{11}(t)=x_{12}(t), \\
& \dot{x}_{12}(t)=x_{13}(t), \\
& \dot{x}_{1\left(n_{1}-1\right)}(t)=x_{1 n_{1}}(t) \text {, } \\
& \dot{x}_{1 n_{1}}(t)=f_{1}(\mathbf{x}(t))+\sum_{j=1}^{p} g_{1 j}(\mathbf{x}(t)) u_{j}(t)+\Delta \phi_{1}(\mathbf{x}(t)), \\
& \dot{x}_{p 1}(t)=x_{p 2}(t), \\
& \dot{x}_{p 2}(t)=x_{p 3}(t), \\
& \dot{x}_{p\left(n_{p}-1\right)}(t)=x_{p n_{p}}(t) \text {, } \\
& \dot{x}_{p n_{p}}(t)=f_{p}(\mathbf{x}(t))+\sum_{j=1}^{P} g_{p j}(\mathbf{x}(t)) u_{j}(t)+\Delta \phi_{p}(\mathbf{x}(t)) \text {, } \\
& y_{1}(t)=x_{11}(t-\tau), \\
& y_{p}(t)=x_{p 1}(t-\tau),
\end{aligned}
$$

or equivalently

$$
\begin{gathered}
\dot{\mathbf{x}}(t)=\mathbf{A} \mathbf{x}(t)+\mathbf{B}[\mathbf{F}(\mathbf{x}(t))+\mathbf{G}(\mathbf{x}(t)) \mathbf{u}(t)+\Delta \mathbf{\Phi}(\mathbf{x}(t))], \\
\mathbf{y}(t)=\mathbf{C} \mathbf{x}(t-\tau),
\end{gathered}
$$

where

$$
\begin{gathered}
\mathbf{F}(\mathbf{x})=\left[f_{1}(\mathbf{x}), \ldots, f_{p}(\mathbf{x})\right]^{T} \in R^{p}, \\
\mathbf{G}(\mathbf{x})=\left[\mathbf{G}_{1}(\mathbf{x}), \ldots, \mathbf{G}_{p}(\mathbf{x})\right] \in R^{p \times p}, \\
\mathbf{G}_{i}(\mathbf{x})=\left[g_{1 i}(\mathbf{x}), \ldots, g_{p i}(\mathbf{x})\right]^{T} \in R^{p}, \quad i=1,2, \ldots, p, \\
\mathbf{A}=\operatorname{diag}\left[\mathbf{A}_{1}, \ldots, \mathbf{A}_{p}\right] \in R^{n \times n}, \\
\mathbf{B}=\operatorname{diag}\left[\mathbf{B}_{1}, \ldots, \mathbf{B}_{p}\right] \in R^{n \times p}, \\
\mathbf{C}=\operatorname{diag}\left[\mathbf{C}_{1}, \ldots, \mathbf{C}_{p}\right] \in R^{p \times n},
\end{gathered}
$$




$$
\begin{gathered}
\mathbf{A}_{i}=\left[\begin{array}{ccccc}
0 & 1 & 0 & \cdots & 0 \\
0 & 0 & 1 & \cdots & 0 \\
\vdots & \vdots & \vdots & \cdots & 1 \\
0 & 0 & 0 & \cdots & 0
\end{array}\right] \in R^{n_{i} \times n_{i}}, \\
\mathbf{B}_{i}=\left[\begin{array}{c}
0 \\
0 \\
\vdots \\
1
\end{array}\right] \in R^{n_{i}}, \\
\end{gathered}
$$

where $\mathbf{x}=\left[x_{11}, x_{12}, \ldots, x_{1 n_{1}}, \ldots, x_{p 1}, x_{p 2}, \ldots, x_{p n_{p}}\right]^{T} \in R^{n}$ is the system state vector which is assumed to be unavailable for measurement, $\mathbf{u}=\left[u_{1}, \ldots, u_{p}\right]^{T} \in R^{p}$ is the control input, $\mathbf{y}=\left[y_{1}, \ldots, y_{p}\right]^{T} \in R^{p}$ is the output vector, $n_{1}+n_{2}+\cdots+n_{p}=$ $n, \tau>0$ is a known time delay of the system, $f_{i}(\mathbf{x}(t))$ and $g_{i j}(\mathbf{x}(t))$ are the unknown nonlinear system functions, and $\Delta \Phi(\mathbf{x}(t))=\left[\Delta \phi_{1}\left(\mathbf{x}(t), \Delta \phi_{2}\left(\mathbf{x}(t), \ldots, \Delta \phi_{p}(\mathbf{x}(t)]^{T} \in R^{p}\right.\right.\right.$ is the vector of uncertainties.

Now let the output of the system and its derivatives be expressed as follows:

$$
\begin{gathered}
y_{i}(t)=x_{i 1}(t-\tau), \\
\dot{y}_{i}(t)=\dot{x}_{i 1}(t-\tau)=x_{i 2}(t-\tau), \\
\vdots \\
y_{i}^{\left(n_{i}-1\right)}(t)=\dot{x}_{i\left(n_{i}-1\right)}(t-\tau)=x_{i n_{i}}(t-\tau), \\
y_{i}^{\left(n_{i}\right)}(t)=\dot{x}_{i n_{i}}(t-\tau) \\
=f_{i}(\mathbf{x}(t-\tau))+\sum_{j=1}^{p} g_{i j}(\mathbf{x}(t-\tau)) u_{j}(t-\tau) \\
+\Delta \phi_{i}(\mathbf{x}(t-\tau)) .
\end{gathered}
$$

Then, let $\mathbf{y}_{d}=\left[y_{1 d}, \ldots, y_{p d}\right]^{T} \in R^{p}$ be the given bounded output desired signal and contain finite derivatives up to the $n$th order. The tracking errors of the system can be defined as $e_{i j}(t)=y_{i}^{(j-1)}(t)-y_{i d}^{(j-1)}(t)$, for $i=1,2, \ldots, p$ and $j=$ $1,2, \ldots, n_{i}$, and its derivatives can be obtained as

$$
\begin{gathered}
\dot{e}_{i 1}(t)=\dot{y}_{i}(t)-\dot{y}_{i d}(t)=e_{i 2}(t), \\
\dot{e}_{i 2}(t)=y_{i}^{(2)}(t)-y_{i d}{ }^{(2)}(t)=e_{i 3}(t), \\
\vdots \\
\dot{e}_{i\left(n_{i}-1\right)}(t)=y_{i}^{\left(n_{i}-1\right)}(t)-y_{i d}{ }^{\left(n_{i}-1\right)}(t)=e_{1 n_{i}}(t),
\end{gathered}
$$

$$
\begin{aligned}
\dot{e}_{i n_{i}}(t)= & y_{i}{ }^{\left(n_{i}\right)}(t)-y_{i d}{ }^{\left(n_{i}\right)}(t) \\
= & f_{i}(\mathbf{x}(t-\tau))+\sum_{j=1}^{p} g_{i j}(\mathbf{x}(t-\tau)) u_{j}(t-\tau) \\
& +\Delta \phi_{i}(\mathbf{x}(t-\tau))-y_{i d}{ }^{\left(n_{i}\right)}(t) \\
= & f_{i}(\mathbf{x}(t-\tau))+\sum_{j=1}^{p} g_{i j}(\mathbf{x}(t-\tau)) u_{j}(t) \\
& -y_{i d}{ }^{\left(n_{i}\right)}(t)+\Delta \psi_{i}(\mathbf{x}, \mathbf{u}),
\end{aligned}
$$

where the uncertainty $\Delta \psi_{i}(\mathbf{x}, \mathbf{u})$ including the structural uncertainty is defined as

$$
\begin{aligned}
\Delta \psi_{i}(\mathbf{x}, \mathbf{u})=\sum_{j=1}^{p} & g_{i j}(\mathbf{x}(t-\tau))\left[u_{j}(t-\tau)-u_{j}(t)\right] \\
& +\Delta \phi_{i}(\mathbf{x}(t-\tau))
\end{aligned}
$$

Thus, (5) can be expressed as

$$
\begin{gathered}
\dot{\mathbf{e}}_{i}(t)=\mathbf{A}_{i} \mathbf{e}_{i}(t)+\mathbf{B}_{i}\left[f_{i}(\mathbf{x}(t-\tau))+\sum_{j=1}^{p} g_{i j}(\mathbf{x}(t-\tau)) u_{j}(t)\right. \\
\left.-y_{i d}^{\left(n_{i}\right)}(t)+\Delta \psi_{i}(\mathbf{x}, \mathbf{u})\right]
\end{gathered}
$$

From (7), it is easy to verify that the tracking error dynamics of the system (2) can be represented in the following form:

$$
\begin{aligned}
\dot{\mathbf{e}}(t)=\mathbf{A e}(t)+\mathbf{B}[\mathbf{F}(\mathbf{x}(t-\tau))+\mathbf{G}(\mathbf{x}(t-\tau)) \mathbf{u}(t) \\
\left.-\underline{\mathbf{Y}_{d}}(t)+\Delta \Psi(\mathbf{x}, \mathbf{u})\right] . \\
\underline{\mathbf{e}_{1}}(t)=\mathbf{C e}(t),
\end{aligned}
$$

where

$$
\begin{aligned}
\mathbf{e} & =\left[\mathbf{e}_{1}^{T}, \mathbf{e}_{2}^{T}, \ldots, \mathbf{e}_{p}^{T}\right]^{T} \\
& =\left[e_{11}, \ldots, e_{1 n_{1}}, \ldots, e_{p 1}, \ldots, e_{p n_{p}}\right]^{T} \in R^{n}, \\
\underline{\mathbf{e}_{1}} & =\left[e_{11}, e_{21}, \ldots, e_{p 1}\right]^{T} \in R^{p}, \\
\Delta \boldsymbol{\Psi} & =\left[\Delta \psi_{1}, \Delta \psi_{2}, \ldots, \Delta \psi_{p}\right]^{T} \in R^{p}, \\
\mathbf{e}_{i} & =\left[e_{i 1}, e_{i 2}, \ldots, e_{i n_{i}}\right]^{T} \in R^{n_{i}}, \\
\underline{\mathbf{Y}_{d}} & =\left[y_{1 d}^{\left(n_{1}\right)}, y_{2 d}^{\left(n_{2}\right)}, \ldots, y_{p d}^{\left(n_{p}\right)}\right]^{T} \in R^{p}, \\
\mathbf{Y}_{d} & =\left[y_{1 d}, \ldots, y_{1 d}^{\left(n_{1}-1\right)}, \ldots, y_{p d}, \ldots, y_{p d}{ }^{\left(n_{p}-1\right)}\right]^{T} \in R^{n} .
\end{aligned}
$$

Based on the above discussion, the following assumptions should be made for the controller design. 
Assumption 1. The matrix $\mathbf{G}(\mathbf{x})$ is nonsingular (i.e., $\mathbf{G}(\mathbf{x})^{-1}$ exists) for all $\mathbf{x} \in U_{x}$ where $U_{x}$ is a compact set in $R^{n}$.

Assumption 2. $\|\Delta \Psi(\mathbf{x}, \mathbf{u})\| \leq h_{1}(\mathbf{x})+h_{2}(\mathbf{x}(t-\tau))$, where $h_{1}(\mathbf{x})$ and $h_{2}(\mathbf{x}(t-\tau))$ are the unknown positive smooth continuous functions.

Remark 3. Compared with the previous results $[27,30]$, the uncertainties including the structural uncertainty are taken into account in this paper. In addition, the uncertainties considered in this paper are not supposed to be a bounded external disturbance unlike the previous results $[17,18,20-$ 23].

The control objective of this paper is to design a control law $\mathbf{u}(t)$ such that $\mathbf{y}(t)$ can follow a given desired reference signal $\mathbf{y}_{m}(t)$ and guarantee that all the signals involved in the whole closed-loop system are bounded.

2.2. Description of Fuzzy Logic Systems. The basic configuration of the fuzzy logic system consists of four main components: fuzzy rule base, fuzzy inference engine, fuzzifier and defuzzifier $[12,16]$. The fuzzy logic system performs a mapping from $U \subset R^{n}$ to $V \subset R$. Let $U=U_{1} \times \cdots \times U_{n}$ where $U_{i} \subset R, i=1,2, \ldots, n$. The fuzzifier maps a crisp point in $U$ into a fuzzy set in $U$. The fuzzy rule base consists of a collection of fuzzy IF-THEN rules:

$$
\begin{aligned}
& R^{(l)}: \text { IF } x_{1} \text { is } F_{1}^{l} \text {, and } x_{2} \text { is } F_{2}^{l}, \ldots, x_{n} \text { is } F_{n}^{l} \\
& \quad \text { THEN } y_{j} \text { is } G_{j}^{l}, \quad j=1, \ldots, p, l=1, \ldots, N,
\end{aligned}
$$

in which $\mathbf{x}=\left[x_{1}, x_{2}, \ldots, x_{n}\right]^{T} \in U$ and $y \in V \subset R$ are the input and output of the fuzzy logic system, $F_{i}^{l}$ and $G_{j}^{l}$ are fuzzy sets in $U_{i}$ and $V_{j}$, respectively. $N$ is the number of rules. The fuzzy inference engine performs a mapping from fuzzy sets in $U$ to fuzzy sets in $V$, based upon the fuzzy IFTHEN rules in the fuzzy rule base and the compositional rule of inference. The defuzzifier maps a fuzzy set in $V$ to a crisp point in $V$. Through singleton fuzzification, center average defuzzification, and product inference, the output of the fuzzy logic system can be expressed as

$$
y_{j}=\theta_{j}^{T} \xi(x), \quad j=1, \ldots, p,
$$

where $\theta_{j}^{T}=\left[\theta_{j}^{1}, \ldots, \theta_{j}^{N}\right]$ with each variable $\theta_{j}^{l}$ as the point at which the fuzzy membership function of $G_{j}^{l}$ achieves the maximum value, and $\xi(x)=\left[\xi_{1}(x), \ldots, \xi_{N}(x)\right]^{T}$ with each variable $\xi_{1}$ as the fuzzy basis function defined as

$$
\xi_{l}(x)=\frac{\prod_{i=1}^{n} \mu_{F_{i}^{l}}\left(x_{i}\right)}{\sum_{l=1}^{N}\left(\prod_{i=1}^{n} \mu_{F_{i}^{l}}\left(x_{i}\right)\right)},
$$

where $\mu_{F_{i}^{l}}\left(x_{i}\right)$ is the membership function of the fuzzy set. follows:

Now let MIMO fuzzy logic systems be expressed as

$$
\mathbf{y}=\Phi(\mathbf{x}) \boldsymbol{\theta}
$$

where

$$
\begin{gathered}
\boldsymbol{\theta}^{T}=\left[\theta_{1}^{T}, \ldots, \theta_{p}^{T}\right], \\
\theta_{i}^{T}=\left[\theta_{i}^{1}, \ldots, \theta_{i}^{N}\right] \quad \text { for } i=1,2, \ldots, p, \\
\Phi(\mathbf{x})=\operatorname{diag}\left[\xi^{T}(\mathbf{x}), \ldots, \xi^{T}(\mathbf{x})\right] .
\end{gathered}
$$

\section{Observer-Based Robust Adaptive Fuzzy Controller Design and Stability Analysis}

According to the description of the fuzzy logic systems presented in Section 2.2, we can construct the following fuzzy logic systems, over a compact set $\Omega_{\mathbf{x}}$, to approximate the unknown nonlinear functions $f_{i}(\mathbf{x}(t))$ and $g_{i j}(\mathbf{x}(t))$ and the unknown upper bounded functions $h_{1}(\mathbf{x}), h_{2}(\mathbf{x}(t-\tau))$ as follows:

$$
\begin{gathered}
\widehat{f}_{i}\left(\mathbf{x} \mid \theta_{f_{1}}\right)=\theta_{f_{i}}^{T} \xi(\mathbf{x}), \quad i=1, \ldots, p, \\
\widehat{g}_{i j}\left(\mathbf{x} \mid \theta_{g_{i j}}\right)=\theta_{g_{i j}}^{T} \xi(\mathbf{x}), \quad i, j=1, \ldots, p, \\
\widehat{h}_{1}(\mathbf{x})=\theta_{h_{1}}^{T} \xi(\mathbf{x}), \\
\widehat{h}_{2}(\mathbf{x}(t-\tau))=\theta_{h_{2}}^{T} \xi(\mathbf{x}(t-\tau)) .
\end{gathered}
$$

Based on the above statements, it can be easily shown that

$$
\begin{aligned}
& \widehat{\mathbf{F}}\left(\mathbf{x} \mid \boldsymbol{\theta}_{1}\right)=\left[\widehat{f}_{1}\left(\mathbf{x} \mid \theta_{f_{1}}\right), \ldots, \widehat{f}_{p}\left(\mathbf{x} \mid \theta_{f_{p}}\right)\right]^{T}=\boldsymbol{\Phi}(\mathbf{x}) \boldsymbol{\theta}_{1}, \\
& \widehat{\mathbf{G}}\left(\mathbf{x} \mid \boldsymbol{\theta}_{2}\right)= {\left[\begin{array}{ccc}
\widehat{g}_{11}\left(\mathbf{x} \mid \theta_{g_{11}}\right), & \ldots, & \widehat{g}_{1 p}\left(\mathbf{x} \mid \theta_{g_{1 p}}\right) \\
\vdots & \ddots & \vdots \\
\widehat{g}_{p 1}\left(\mathbf{x} \mid \theta_{g_{p_{1}}}\right), & \ldots, & \widehat{g}_{p p}\left(\mathbf{x} \mid \theta_{g_{p p}}\right)
\end{array}\right] } \\
&= \boldsymbol{\Phi}(\mathbf{x}) \boldsymbol{\theta}_{2},
\end{aligned}
$$

where

$$
\begin{aligned}
& \boldsymbol{\theta}_{1}^{T}=\left[\theta_{f 1}^{T}, \ldots, \theta_{f p}^{T}\right], \quad \theta_{f i}^{T}=\left[\theta_{f i}^{1}, \ldots, \theta_{f i}^{N}\right], \\
& \boldsymbol{\theta}_{2}=\left[\begin{array}{ccc}
\theta_{g_{11}}, \ldots, & \theta_{g_{1 p}} \\
\vdots & \ddots & \vdots \\
\theta_{g_{p 1}}, \ldots, & \theta_{g_{p p}}
\end{array}\right], \quad \theta_{g_{i j}}^{T}=\left[\theta_{g_{i j}}^{1}, \ldots, \theta_{g_{i j}^{N}}^{N}\right] \text {, } \\
& \theta_{h_{1}}^{T}=\left[\theta_{h_{1}}^{1}, \ldots, \theta_{h_{1}}^{N}\right], \quad \theta_{h_{2}}^{T}=\left[\theta_{h_{2}}^{1}, \ldots, \theta_{h_{2}}^{N}\right] \text {, } \\
& \boldsymbol{\Phi}(\mathbf{x})=\operatorname{diag}\left[\xi^{T}(\mathbf{x}), \ldots, \xi^{T}(\mathbf{x})\right] .
\end{aligned}
$$

Suppose the state variables of the controlled system (1) are available for measurement, then the following robust adaptive fuzzy controller $\mathbf{u}(t)$ can be adopted to let the system achieve the above control objective and can be defined as follows:

$$
\begin{aligned}
\mathbf{u}(t)=\widehat{\mathbf{G}}(\mathbf{x}(t-\tau))^{-1}[ & -\widehat{\mathbf{F}}(\mathbf{x}(t-\tau))-\mathbf{K}_{c} \mathbf{e}(t) \\
& \left.+\underline{\mathbf{Y}_{d}}(t)+\mathbf{u}_{s}(t)\right],
\end{aligned}
$$


where $\mathbf{K}_{c}=\operatorname{diag}\left[\mathbf{K}_{c 1}, \ldots, \mathbf{K}_{c p}\right] \in R^{p \times n}$, where $\mathbf{K}_{c i}=$ $\left[k_{i 1}^{c}, \ldots, k_{i n_{i}}^{c}\right] \in R^{1 \times n_{i}}$ for $i=1,2, \ldots, p$, is the control gain matrix such that the characteristic polynomial of $\mathbf{A}-\mathbf{B K}_{c}$ is Hurwitz. The robust compensator $\mathbf{u}_{s}(t)$ will be designed to compensate the fuzzy approximation errors and the uncertainties.

However, the state variables of the system are unavailable for measurement in many engineering systems. In addition, although the tracking error vector $\mathbf{e}$ can be obtained ideally by successive differentiation of $e_{i 1}(i=1,2, \ldots, p)$, ideal differentiators are physically unrealizable. Obviously, we must employ a state observer to estimate $\mathbf{x}$ and $\mathbf{e}$ and let $\widehat{\mathbf{x}}$ and $\widehat{\mathbf{e}}$ be the estimates of $\mathbf{x}$ and $\mathbf{e}$, respectively. Then replacing $\widehat{\mathbf{F}}\left(\mathbf{x} \mid \boldsymbol{\theta}_{1}\right)$, $\widehat{\mathbf{G}}\left(\mathbf{x} \mid \boldsymbol{\theta}_{2}\right), \widehat{h}_{1}\left(\mathbf{x} \mid \boldsymbol{\theta}_{h}\right), \widehat{h}_{2}(\mathbf{x}(t-\tau)), \mathbf{x}$, and e by $\widehat{\mathbf{F}}\left(\widehat{\mathbf{x}} \mid \boldsymbol{\theta}_{1}\right)$, $\widehat{\mathbf{G}}\left(\widehat{\mathbf{x}} \mid \boldsymbol{\theta}_{2}\right), \widehat{h}_{1}\left(\widehat{\mathbf{x}} \mid \boldsymbol{\theta}_{h}\right), \widehat{h}_{2}(\widehat{\mathbf{x}}(t-\tau)), \widehat{\mathbf{x}}$, and $\widehat{\mathbf{e}}$, respectively, the robust adaptive fuzzy controller (18) cannot be used to control the nonlinear system (2) and will be modified as the following form:

$$
\begin{array}{r}
\mathbf{u}(t)=\widehat{\mathbf{G}}(\widehat{\mathbf{x}}(t-\tau))^{-1}\left[-\widehat{\mathbf{F}}(\widehat{\mathbf{x}}(t-\tau))-\mathbf{K}_{c} \widehat{\mathbf{e}}(t)\right. \\
\left.+\underline{\mathbf{Y}_{d}}(t)+\mathbf{u}_{s}(t)\right],
\end{array}
$$

where

$$
\begin{gathered}
\widehat{\mathbf{F}}\left(\widehat{\mathbf{x}} \mid \boldsymbol{\theta}_{1}\right)=\left[\widehat{f}_{1}\left(\widehat{\mathbf{x}} \mid \theta_{f_{1}}\right), \ldots, \widehat{f}_{p}\left(\widehat{\mathbf{x}} \mid \theta_{f_{p}}\right)\right]^{T}=\boldsymbol{\Phi}(\widehat{\mathbf{x}}) \boldsymbol{\theta}_{1}, \\
\widehat{\mathbf{G}}\left(\widehat{\mathbf{x}} \mid \boldsymbol{\theta}_{2}\right)=\left[\begin{array}{ccc}
\widehat{g}_{11}\left(\widehat{\mathbf{x}} \mid \theta_{g_{11}}\right), & \ldots, & \widehat{g}_{1 p}\left(\widehat{\mathbf{x}} \mid \theta_{g_{1 p}}\right) \\
\vdots & \ddots & \vdots \\
\widehat{g}_{p 1}\left(\widehat{\mathbf{x}} \mid \theta_{g_{p_{1}}}\right), & \ldots, & \widehat{g}_{p p}\left(\widehat{\mathbf{x}} \mid \theta_{g_{p p}}\right)
\end{array}\right] \\
=\boldsymbol{\Phi ( \widehat { \mathbf { x } } ) \boldsymbol { \theta } _ { 2 } ,} \\
\widehat{h}_{1}(\widehat{\mathbf{x}})=\theta_{h_{1}}^{T} \xi(\widehat{\mathbf{x}}) ; \quad \widehat{h}_{2}(\widehat{\mathbf{x}}(t-\tau))=\theta_{h_{2}}^{T} \xi(\widehat{\mathbf{x}}(t-\tau)) .
\end{gathered}
$$

Substituting (19) into (8) yields

$$
\begin{aligned}
& \dot{\mathbf{e}}(t)=\mathbf{A e}(t)-\mathbf{B K}_{c} \widehat{\mathbf{e}}(t) \\
&+\mathbf{B}\{ \mathbf{F}(\mathbf{x}(t-\tau))-\widehat{\mathbf{F}}\left(\widehat{\mathbf{x}}(t-\tau) \mid \boldsymbol{\theta}_{1}\right) \\
&+ {\left[\mathbf{G}(\mathbf{x}(t-\tau))-\widehat{\mathbf{G}}\left(\widehat{\mathbf{x}}(t-\tau) \mid \boldsymbol{\theta}_{2}\right)\right] \mathbf{u}(t) } \\
&+\left.\mathbf{u}_{s}(t)+\Delta \boldsymbol{\Psi}(\mathbf{x}, \mathbf{u})\right\}, \\
& \underline{\mathbf{e}}_{1}(t)=\mathbf{C e}(t) .
\end{aligned}
$$

In order to estimate the output tracking error vector, we design the observer as follows:

$$
\begin{gathered}
\dot{\hat{\mathbf{e}}}(t)=\left(\mathbf{A}-\mathbf{B} \mathbf{K}_{c}\right) \widehat{\mathbf{e}}(t)+\mathbf{K}_{o}\left(\underline{\mathbf{e}_{1}}(t)-\underline{\widehat{\mathbf{e}}_{1}}(t)\right), \\
\underline{\widehat{\mathbf{e}}_{1}}(t)=\mathbf{C} \widehat{\mathbf{e}}(t),
\end{gathered}
$$

where $\mathbf{K}_{o}=\operatorname{diag}\left[\mathbf{K}_{o 1}, \ldots, \mathbf{K}_{o p}\right] \in R^{n \times p}$, where $\mathbf{K}_{o i}=$ $\left[k_{1 i}^{o}, \ldots, k_{n_{i} i}^{o}\right]^{T}$ for $i=1,2, \ldots, p$, is the observer gain matrix such that the characteristic polynomial of $\mathbf{A}-\mathbf{K}_{o} \mathbf{C}$ is Hurwitz. Let us define the observation error vector as

$$
\widetilde{\mathbf{e}}(t)=\mathbf{e}(t)-\widehat{\mathbf{e}}(t) .
$$

Then by (23) and (24), we obtain

$$
\begin{aligned}
& \dot{\tilde{\mathbf{e}}}(t)=\left(\mathbf{A}-\mathbf{K}_{o} \mathbf{C}\right) \widetilde{\mathbf{e}}(t) \\
&+\mathbf{B}\left\{\mathbf{F}(\mathbf{x}(t-\tau))-\widehat{\mathbf{F}}\left(\widehat{\mathbf{x}}(t-\tau) \mid \boldsymbol{\theta}_{1}\right)\right. \\
&+\left[\mathbf{G}(\mathbf{x}(t-\tau))-\widehat{\mathbf{G}}\left(\widehat{\mathbf{x}}(t-\tau) \mid \boldsymbol{\theta}_{2}\right)\right] \mathbf{u}(t) \\
&\left.+\mathbf{u}_{\mathbf{s}}(t)+\Delta \boldsymbol{\Psi}(\mathbf{x}, \mathbf{u})\right\}, \\
& \widetilde{\mathbf{e}}_{1}(t)=\mathbf{C} \widetilde{\mathbf{e}}(t) .
\end{aligned}
$$

It is assumed that $\boldsymbol{\theta}_{1}, \boldsymbol{\theta}_{2}, \theta_{h_{1}}$, and $\theta_{h_{2}}$ belong to compact sets $\Omega_{\theta_{1}}, \Omega_{\theta_{2}}, \Omega_{\theta_{h_{1}}}$, and $\Omega_{\theta_{h_{2}}}$, respectively, which are defined as

$$
\begin{array}{r}
\Omega_{\theta_{1}}=\left\{\boldsymbol{\theta}_{1} \in R^{N \times p}:\left\|\boldsymbol{\theta}_{1}\right\| \leq M_{\boldsymbol{\theta}_{1}}<\infty\right\}, \\
\Omega_{\boldsymbol{\theta}_{2}}=\left\{\boldsymbol{\theta}_{2} \in R^{p N \times p}:\left\|\boldsymbol{\theta}_{2}\right\| \leq M_{\boldsymbol{\theta}_{2}}<\infty\right\}, \\
\Omega_{\theta_{h_{1}}}=\left\{\theta_{h_{1}} \in R^{N}:\left\|\theta_{h_{1}}\right\| \leq M_{\theta_{h_{1}}}<\infty\right\}, \\
\Omega_{\theta_{h_{2}}}=\left\{\theta_{h_{2}} \in R^{N}:\left\|\theta_{h_{2}}\right\| \leq M_{\theta_{h_{2}}}<\infty\right\},
\end{array}
$$

where $M_{\boldsymbol{\theta}_{1}}, M_{\boldsymbol{\theta}_{2}}, M_{\theta_{h_{1}}}$, and $M_{\theta_{h_{2}}}$ are the designed parameters by the designer, and $N$ is the number of fuzzy inference rules.

Now let us define the optimal parameter vectors $\boldsymbol{\theta}_{1}^{*}, \boldsymbol{\theta}_{2}^{*}$, $\theta_{h_{1}}^{*}$, and $\theta_{h_{2}}^{*}$ as follows:

$$
\begin{gathered}
\boldsymbol{\theta}_{1}^{*}=\arg \min _{\boldsymbol{\theta}_{1} \in \Omega_{\theta_{1}}}\left\{\sup _{\mathbf{x} \in \Omega_{\mathbf{x}}, \widehat{\mathbf{x}} \in \Omega_{\widehat{\mathbf{x}}}}\left|\mathbf{F}(\mathbf{x})-\widehat{\mathbf{F}}\left(\widehat{\mathbf{x}} \mid \boldsymbol{\theta}_{1}\right)\right|\right\}, \\
\boldsymbol{\theta}_{2}^{*}=\arg \min _{\boldsymbol{\theta}_{2} \in \Omega_{\theta_{2}}}\left\{\sup _{\mathbf{x} \in \Omega_{\mathbf{x}}, \widehat{\mathbf{x}} \in \Omega_{\overline{\mathbf{x}}}}\left|\mathbf{G}(\mathbf{x})-\widehat{\mathbf{G}}\left(\widehat{\mathbf{x}} \mid \boldsymbol{\theta}_{2}\right)\right|\right\}, \\
\theta_{h_{1}}^{*}=\arg \min _{\boldsymbol{\theta}_{h_{1}} \in \Omega_{\theta_{h_{1}}}}\left\{\sup _{\mathbf{x} \in \Omega_{\mathbf{x}}, \widehat{\mathbf{x}} \in \Omega_{\overline{\mathbf{x}}}}\left|h_{1}(\mathbf{x})-\widehat{h}_{1}\left(\widehat{\mathbf{x}} \mid \theta_{h_{1}}\right)\right|\right\}, \\
\theta_{h_{2}}^{*}=\arg \min _{\boldsymbol{\theta}_{h_{2}} \in \Omega_{\theta_{h 2}}}\left\{\sup _{\mathbf{x} \in \Omega_{\mathbf{x}}, \widehat{\mathbf{x}} \in \Omega_{\overline{\mathbf{x}}}} \mid h_{2}(\mathbf{x}(t-\tau))\right. \\
\left.\left.-\widehat{h}_{2}(\widehat{\mathbf{x}}(t-\tau)) \mid \theta_{h_{2}}\right) \mid\right\},
\end{gathered}
$$

where $\boldsymbol{\theta}_{1}^{*}, \boldsymbol{\theta}_{2}^{*}, \theta_{h_{1}}^{*}$, and $\theta_{h_{2}}^{*}$ are bounded in the suitable closed sets $\Omega_{\theta_{1}}, \Omega_{\theta_{2}}, \Omega_{\theta_{h_{1}}}$, and $\Omega_{\theta_{h_{2}}}$, respectively. Also the parameter estimation errors are defined as

$$
\begin{gathered}
\tilde{\boldsymbol{\theta}}_{1}=\boldsymbol{\theta}_{1}^{*}-\boldsymbol{\theta}_{1}, \\
\tilde{\boldsymbol{\theta}}_{2}=\boldsymbol{\theta}_{2}^{*}-\boldsymbol{\theta}_{2}, \\
\widetilde{\theta}_{h_{1}}=\theta_{h_{1}}^{*}-\theta_{h_{1}} ; \quad \widetilde{\theta}_{h_{2}}=\theta_{h_{2}}^{*}-\theta_{h_{2}} .
\end{gathered}
$$


Then, the minimum approximation errors which correspond to the optimal parameter vectors are defined as

$$
\begin{aligned}
\boldsymbol{\omega}_{1}= & \mathbf{F}(\mathbf{x}(t-\tau))-\widehat{\mathbf{F}}\left(\widehat{\mathbf{x}}(t-\tau) \mid \boldsymbol{\theta}_{1}^{*}\right) \\
& +\left(\mathbf{G}(\mathbf{x}(t-\tau))-\widehat{\mathbf{G}}\left(\widehat{\mathbf{x}}(t-\tau) \mid \boldsymbol{\theta}_{2}^{*}\right)\right) \mathbf{u}, \\
\omega_{2}= & h_{1}(\mathbf{x})-\widehat{h}_{1}\left(\widehat{\mathbf{x}} \mid \theta_{h_{1}}^{*}\right) \\
& +h_{2}(\mathbf{x}(t-\tau))-\widehat{h}_{2}\left(\widehat{\mathbf{x}}(t-\tau) \mid \theta_{h_{2}}^{*}\right) .
\end{aligned}
$$

Applying (29), (30), and (32), (26) can be rewritten as

$$
\begin{aligned}
& \dot{\overrightarrow{\mathbf{e}}}(t)=\left(\mathbf{A}-\mathbf{K}_{o} \mathbf{C}\right) \widetilde{\mathbf{e}}(t) \\
&+\mathbf{B}\left\{\boldsymbol{\Phi}(\widehat{\mathbf{x}}) \widetilde{\boldsymbol{\theta}}_{1}+\boldsymbol{\Phi}(\widehat{\mathbf{x}}) \widetilde{\boldsymbol{\theta}}_{2} \mathbf{u}(t)\right.+\mathbf{F}(\mathbf{x}(t-\tau))-\widehat{\mathbf{F}}\left(\widehat{\mathbf{x}}(t-\tau) \mid \boldsymbol{\theta}_{1}^{*}\right) \\
&+\left[\mathbf{G}(\mathbf{x}(t-\tau))-\widehat{\mathbf{G}}\left(\widehat{\mathbf{x}}(t-\tau) \mid \boldsymbol{\theta}_{2}^{*}\right)\right] \mathbf{u}(t) \\
&\left.+\mathbf{u}_{s}(t)+\Delta \boldsymbol{\Psi}(\mathbf{x}, \mathbf{u})\right\} \\
&=\left(\mathbf{A}-\mathbf{K}_{o} \mathbf{C}\right) \widetilde{\mathbf{e}}(t)+\mathbf{B}\left[\boldsymbol{\Phi}(\widehat{\mathbf{x}}) \widetilde{\boldsymbol{\theta}}_{1}+\boldsymbol{\Phi}(\widehat{\mathbf{x}}) \widetilde{\boldsymbol{\theta}}_{2} \mathbf{u}(t)\right.\left.+\boldsymbol{\omega}_{1}+\mathbf{u}_{s}(t)+\Delta \Psi(\mathbf{x}, \mathbf{u})\right], \\
& \widetilde{\mathbf{e}}_{1}(t)=\mathbf{C} \widetilde{\mathbf{e}}(t) .
\end{aligned}
$$

Then, the output error dynamics in (34) can be expressed as follows:

$$
\begin{aligned}
\underline{\tilde{\mathbf{e}}_{1}}=\mathbf{H}(\mathbf{s})\left[\boldsymbol{\Phi}(\widehat{\mathbf{x}}) \tilde{\boldsymbol{\theta}}_{1}+\boldsymbol{\Phi}(\widehat{\mathbf{x}}) \tilde{\boldsymbol{\theta}}_{2} \mathbf{u}(t)+\omega_{1}+\mathbf{u}_{s}(t)\right. \\
+\Delta \boldsymbol{\Psi}(\mathbf{x}, \mathbf{u})],
\end{aligned}
$$

where

$$
\mathbf{H}(\mathbf{s})=\mathbf{C}\left(\mathbf{s I}-\left(\mathbf{A}-\mathbf{K}_{o} \mathbf{C}\right)\right)^{-1} \mathbf{B} .
$$

Obviously, the transfer function $\mathbf{H}(\mathbf{s})$ is a known stable transfer function matrix. In order to utilize the SPR-Lyapunov design approach, (35) can be represented as

$$
\begin{aligned}
& \underline{\tilde{\mathbf{e}}_{1}}=\mathbf{H}(\mathbf{s}) \mathbf{L}(\mathbf{s})\left[\mathbf{L}(\mathbf{s})^{-1} \boldsymbol{\Phi}(\widehat{\mathbf{x}}) \widetilde{\boldsymbol{\theta}}_{1}+\mathbf{L}(\mathbf{s})^{-1} \boldsymbol{\Phi}(\widehat{\mathbf{x}}) \widetilde{\boldsymbol{\theta}}_{2} \mathbf{u}(t)\right. \\
& \left.+\mathbf{L}(\mathbf{s})^{-1}\left(\boldsymbol{\omega}_{1}+\mathbf{u}_{s}(t)+\Delta \Psi(\mathbf{x}, \mathbf{u})\right)\right],
\end{aligned}
$$

with

$$
\begin{gathered}
\mathbf{L}(s)=\operatorname{diag}\left[L_{i}(s), \ldots, L_{p}(s)\right], \\
L_{i}(s)=s^{m_{i}}+b_{i 1} s^{m_{i}-1}+\cdots+b_{i m_{i}} \\
\quad\left(m_{i}=n_{i}-1\right), i=1,2, \ldots, p .
\end{gathered}
$$

It is worth noting that $\mathbf{L}(s)$ is chosen to be a proper stable transfer function matrix and to make $\mathbf{H}(\mathbf{s}) \mathbf{L}(\mathbf{s})$ to be a proper
SPR transfer function matrix. Then the state-space realization of (37) can be written as

$$
\begin{aligned}
\dot{\tilde{\mathbf{e}}}_{c}(t)=\left(\mathbf{A}-\mathbf{K}_{o} \mathbf{C}\right) \widetilde{\mathbf{e}}_{c}+\mathbf{B}_{c}[ & \boldsymbol{\Phi}_{f}(\widehat{\mathbf{x}}) \widetilde{\boldsymbol{\theta}}_{1}+\boldsymbol{\Phi}_{f}(\widehat{\mathbf{x}}) \widetilde{\boldsymbol{\theta}}_{2} \mathbf{u}(t) \\
& \left.+\boldsymbol{\omega}_{1 f}+\mathbf{u}_{s f}(t)+\Delta \boldsymbol{\Psi}_{f}(\mathbf{x}, \mathbf{u})\right], \\
\widetilde{\mathbf{e}}_{1}(t)= & \mathbf{C} \widetilde{\mathbf{e}}_{c}(t),
\end{aligned}
$$

with

$$
\begin{gathered}
\mathbf{B}_{c}=\operatorname{diag}\left[\mathbf{B}_{c 1}, \ldots, \mathbf{B}_{c p}\right] \in R^{n \times p} \\
\mathbf{B}_{c i}=\left[1, b_{i 1}, b_{i 2}, \ldots, b_{i m_{i}}\right]^{T} \in R^{n_{i} \times 1}, \quad i=1,2, \ldots, p \\
\boldsymbol{\Phi}_{f}(\widehat{\mathbf{x}})=\mathbf{L}(\mathbf{s})^{-1} \mathbf{\Phi}(\widehat{\mathbf{x}}) \\
\boldsymbol{\omega}_{1 f}=\mathbf{L}(\mathbf{s})^{-1} \boldsymbol{\omega}_{1} \\
\mathbf{u}_{s f}=\mathbf{L}(\mathbf{s})^{-1} \mathbf{u}_{s} \\
\Delta \Psi_{f}(\mathbf{x}, \mathbf{u})=\mathbf{L}(\mathbf{s})^{-1} \Delta \Psi(\mathbf{x}, \mathbf{u}) .
\end{gathered}
$$

We define

$$
\begin{gathered}
|| \omega_{1 f} \|+\left|\omega_{2}\right| \leq \omega_{f}, \\
\widetilde{\omega}_{f}=\omega_{f}-\widehat{\omega}_{f},
\end{gathered}
$$

where $\widehat{\omega}_{f}$ is the estimate of an unknown positive constant $\omega_{f}$.

Remark 4. Since $\mathbf{L}(\mathbf{s})$ is chosen as a proper stable transfer function matrix, it is obvious that the norm of $\Delta \Psi_{f}(\mathbf{x}, \mathbf{u})$ also satisfies the inequality in Assumption 2. Moreover, the upper bound functions will be replaced by $\widehat{h}_{1}\left(\widehat{\mathbf{x}} \mid \boldsymbol{\theta}_{h}\right)$ and $\widehat{h}_{2}(\widehat{\mathbf{x}}(t-\tau))$ because the state variables are unavailable for measurement.

Based on the Lyapunov stability theorem, we can obtain the robust compensator $\mathbf{u}_{s f}(t)$ as follows:

$$
\mathbf{u}_{s f}(t)=\mathbf{u}_{1}(t)+\mathbf{u}_{2}(t),
$$

where

$$
\begin{aligned}
\mathbf{u}_{1}(t)=- & \frac{\mathbf{B}_{c}^{T} \mathbf{P}_{1} \widetilde{\mathbf{e}}_{c}}{\left\|\widetilde{\mathbf{e}}_{c}^{T} \mathbf{P}_{1} \mathbf{B}_{c}\right\|} \\
& \times\left[\widehat{\omega}_{f}+\widehat{h}_{1}\left(\widehat{\mathbf{x}} \mid \theta_{h_{1}}\right)+\widehat{h}_{2}\left(\widehat{\mathbf{x}}(t-\tau) \mid \theta_{h_{2}}\right)\right], \\
\mathbf{u}_{2}(t)=- & \frac{\mathbf{B}_{c}^{T} \mathbf{P}_{1} \widetilde{\mathbf{e}}_{c}}{\left\|\widetilde{\mathbf{e}}_{c}^{T} \mathbf{P}_{1} \mathbf{B}_{c}\right\|^{2}}\left[\widehat{\mathbf{e}}^{T} \mathbf{P}_{2} \mathbf{K}_{o} \underline{\widetilde{\mathbf{e}}_{1}}\right],
\end{aligned}
$$

where $\mathbf{K}_{c}$ is the control gain matrix, $\mathbf{K}_{o}$ is the observer gain matrix, and $\mathbf{P}_{1}$ and $\mathbf{P}_{2}$ are the symmetric positive definite matrices and will be solved later.

Then, the parameter adaptive learning laws are chosen as

$$
\begin{gathered}
\dot{\boldsymbol{\theta}}_{1}=\gamma_{1} \boldsymbol{\Phi}_{f}^{T}(\widehat{\mathbf{x}}) \mathbf{B}_{c}^{T} \mathbf{P}_{1} \widetilde{\mathbf{e}}_{c}, \\
\dot{\boldsymbol{\theta}}_{2}=\gamma_{2} \boldsymbol{\Phi}_{f}^{T}(\widehat{\mathbf{x}}) \mathbf{B}_{c}^{T} \mathbf{P}_{1} \widetilde{\mathbf{e}}_{c} \mathbf{u}^{T},
\end{gathered}
$$




$$
\begin{gathered}
\dot{\theta}_{h_{1}}=\gamma_{3}\left\|\widetilde{\mathbf{e}}_{c}^{T} \mathbf{P}_{1} \mathbf{B}_{c}\right\| \boldsymbol{\xi}(\widehat{\mathbf{x}}), \\
\dot{\theta}_{h_{2}}=\gamma_{4}\left\|\widetilde{\mathbf{e}}_{c}^{T} \mathbf{P}_{1} \mathbf{B}_{c}\right\| \boldsymbol{\xi}(\widehat{\mathbf{x}}(t-\tau)), \\
\dot{\widehat{\omega}}_{f}=\gamma_{5}\left\|\widetilde{\mathbf{e}}_{c}^{T} \mathbf{P}_{1} \mathbf{B}_{c}\right\|,
\end{gathered}
$$

where $\gamma_{1}>0, \gamma_{2}>0, \gamma_{3}>0, \gamma_{4}>0$, and $\gamma_{5}>0$ are the positive adaptive gain constants and can be chosen by the designer.

Remark 5. Without loss of generality, the adaptive laws used in this paper are assumed such that the parameter vectors are within the constraint sets or on the boundaries of the constraint sets but moving toward the inside of the constraint sets. If the parameter vectors are on the boundaries of the constraint sets but moving toward the outside of the constraint sets, we have to use the projection algorithm [12] to modify the adaptive laws such that the parameter vectors will remain inside of the constraint sets. The proposed adaptive laws (46)-(49) can be modified as the following form:

$$
\dot{\boldsymbol{\theta}}_{1}=\left\{\begin{array}{lc}
\gamma_{1} \boldsymbol{\Phi}_{f}^{T}(\widehat{\mathbf{x}}) \mathbf{B}_{c}^{T} \mathbf{P}_{1} \widetilde{\mathbf{e}}_{c}, & \text { if }\left(\left\|\boldsymbol{\theta}_{1}\right\|<M_{\theta_{1}}\right) \\
& \text { or }\left(\left\|\boldsymbol{\theta}_{1}\right\|=M_{\theta_{1}},\right. \\
& \left.\boldsymbol{\theta}_{1}^{T} \boldsymbol{\Phi}_{f}^{T}(\widehat{\mathbf{x}}) \mathbf{B}_{c}^{T} \mathbf{P}_{1} \widetilde{\mathbf{e}}_{c} \leq 0\right), \\
P\left\{\gamma_{1} \boldsymbol{\Phi}_{f}^{T}(\widehat{\mathbf{x}}) \mathbf{B}_{c}^{T} \mathbf{P}_{1} \widetilde{\mathbf{e}}_{c}\right\}, & \text { if }\left(\left\|\boldsymbol{\theta}_{1}\right\|=M_{\theta_{1}},\right. \\
& \left.\boldsymbol{\theta}_{1}^{T} \boldsymbol{\Phi}_{f}^{T}(\widehat{\mathbf{x}}) \mathbf{B}_{c}^{T} \mathbf{P}_{1} \widetilde{\mathbf{e}}_{c}>0\right),
\end{array}\right.
$$

where $P\left\{\gamma_{1} \boldsymbol{\Phi}_{f}^{T}(\widehat{\mathbf{x}}) \mathbf{B}_{c}^{T} \mathbf{P}_{1} \widetilde{\mathbf{e}}_{c}\right\}$ is defined as

$$
\begin{gathered}
P\left\{\gamma_{1} \boldsymbol{\Phi}_{f}^{T}(\widehat{\mathbf{x}}) \mathbf{B}_{c}^{T} \mathbf{P}_{1} \widetilde{\mathbf{e}}_{c}\right\}=\gamma_{1} \boldsymbol{\Phi}_{f}^{T}(\widehat{\mathbf{x}}) \mathbf{B}_{c}^{T} \mathbf{P}_{1} \widetilde{\mathbf{e}}_{c} \\
-\gamma_{1} \frac{\boldsymbol{\theta}_{1} \boldsymbol{\theta}_{1}^{T}}{\left\|\boldsymbol{\theta}_{1}\right\|^{2}} \boldsymbol{\Phi}_{f}^{T}(\widehat{\mathbf{x}}) \mathbf{B}_{c}^{T} \mathbf{P}_{1} \widetilde{\mathbf{e}}_{c}, \\
\dot{\boldsymbol{\theta}}_{2}=\left\{\begin{array}{cc}
\gamma_{2} \boldsymbol{\Phi}_{f}^{T}(\widehat{\mathbf{x}}) \mathbf{B}_{c}^{T} \mathbf{P}_{1} \widetilde{\mathbf{e}}_{c} \mathbf{u}^{T}, & \text { if }\left(\left\|\boldsymbol{\theta}_{2}\right\|<M_{\theta_{2}}\right) \\
& \text { or }\left(\left\|\boldsymbol{\theta}_{2}\right\|=M_{\theta_{2}},\right. \\
\left.\boldsymbol{\theta}_{2}^{T} \boldsymbol{\Phi}_{f}^{T}(\widehat{\mathbf{x}}) \mathbf{B}_{c}^{T} \mathbf{P}_{1} \widetilde{\mathbf{e}}_{c} \mathbf{u}^{T} \leq 0\right), \\
P\left\{\gamma_{2} \boldsymbol{\Phi}_{f}^{T}(\widehat{\mathbf{x}}) \mathbf{B}_{c}^{T} \mathbf{P}_{1} \widetilde{\mathbf{e}}_{c} \mathbf{u}^{T}\right\}, & \text { if }\left(\left\|\boldsymbol{\theta}_{2}\right\|=M_{\theta_{2}},\right. \\
\left.\boldsymbol{\theta}_{2}^{T} \boldsymbol{\Phi}_{f}^{T}(\widehat{\mathbf{x}}) \mathbf{B}_{c}^{T} \mathbf{P}_{1} \widetilde{\mathbf{e}}_{c} \mathbf{u}^{T}>0\right),
\end{array}\right.
\end{gathered}
$$

where $P\left\{\gamma_{2} \boldsymbol{\Phi}_{f}^{T}(\widehat{\mathbf{x}}) \mathbf{B}_{c}^{T} \mathbf{P}_{1} \widetilde{\mathbf{e}}_{c} \mathbf{u}^{T}\right\}$ is defined as

$$
\begin{aligned}
& P\left\{\gamma_{2} \Phi_{f}^{T}(\widehat{\mathbf{x}}) \mathbf{B}_{c}^{T} \mathbf{P}_{1} \widetilde{\mathbf{e}}_{c} \mathbf{u}^{T}\right\}=\gamma_{2} \boldsymbol{\Phi}_{f}^{T}(\widehat{\mathbf{x}}) \mathbf{B}_{c}^{T} \mathbf{P}_{1} \widetilde{\mathbf{e}}_{c} \mathbf{u}^{T} \\
& -\gamma_{2} \frac{\boldsymbol{\theta}_{2} \boldsymbol{\theta}_{2}^{T}}{\left\|\boldsymbol{\theta}_{2}\right\|^{2}} \boldsymbol{\Phi}_{f}^{T}(\widehat{\mathbf{x}}) \mathbf{B}_{c}^{T} \mathbf{P}_{1} \widetilde{\mathbf{e}}_{c} \mathbf{u}^{T}, \\
& \dot{\theta}_{h_{1}}=\left\{\begin{array}{cc}
\gamma_{3}\left\|\tilde{\mathbf{e}}_{c}^{T} \mathbf{P}_{1} \mathbf{B}_{c}\right\| \boldsymbol{\xi}(\widehat{\mathbf{x}}), & \text { if }\left(\left\|\theta_{h_{1}}\right\|<M_{\theta_{h_{1}}}\right) \\
& \text { or }\left(\left\|\theta_{h_{1}}\right\|=M_{\theta_{h_{1}}},\right. \\
& \left.\left\|\widetilde{\mathbf{e}}_{c}^{T} \mathbf{P}_{1} \mathbf{B}_{c}\right\| \cdot \theta_{h_{1}}^{T} \boldsymbol{\xi}(\widehat{\mathbf{x}}) \leq 0\right), \\
P\left\{\gamma_{3}\left\|\tilde{\mathbf{e}}_{c}^{T} \mathbf{P}_{1} \mathbf{B}_{c}\right\| \boldsymbol{\xi}(\widehat{\mathbf{x}})\right\}, & \text { if }\left(\left\|\theta_{h_{1}}\right\|=M_{\theta_{h_{1}}},\right. \\
\left.\left\|\tilde{\mathbf{e}}_{c}^{T} \mathbf{P}_{1} \mathbf{B}_{c}\right\| \cdot \theta_{h_{1}}^{T} \boldsymbol{\xi}(\widehat{\mathbf{x}})>0\right),
\end{array}\right.
\end{aligned}
$$

where $P\left\{\gamma_{3}\left\|\widetilde{\mathbf{e}}_{c}^{T} \mathbf{P}_{1} \mathbf{B}_{c}\right\| \boldsymbol{\xi}(\widehat{\mathbf{x}})\right\}$ is defined as

$$
\begin{aligned}
& P\left\{\gamma_{3}\left\|\widetilde{\mathbf{e}}_{c}^{T} \mathbf{P}_{1} \mathbf{B}_{c}\right\| \boldsymbol{\xi}(\widehat{\mathbf{x}})\right\}=\gamma_{3}\left\|\widetilde{\mathbf{e}}_{c}^{T} \mathbf{P}_{1} \mathbf{B}_{c}\right\| \boldsymbol{\xi}(\widehat{\mathbf{x}}) \\
& -\gamma_{3} \frac{\theta_{h_{1}} \theta_{h_{1}}^{T}}{\left\|\theta_{h_{1}}\right\|^{2}}\left\|\tilde{\mathbf{e}}_{c}^{T} \mathbf{P}_{1} \mathbf{B}_{c}\right\| \xi(\widehat{\mathbf{x}}), \\
& \dot{\theta}_{h_{2}}=\left\{\begin{array}{cc}
\gamma_{4}\left\|\widetilde{\mathbf{e}}_{c}^{T} \mathbf{P}_{1} \mathbf{B}_{c}\right\| \boldsymbol{\xi}(\widehat{\mathbf{x}}(t-\tau)), & \text { if }\left(\left\|\theta_{h_{2}}\right\|<M_{\theta_{h_{2}}}\right) \\
& \text { or }\left(\left\|\theta_{h_{2}}\right\|=M_{\theta_{h_{2}}},\right. \\
& \left\|\widetilde{\mathbf{e}}_{c}^{T} \mathbf{P}_{1} \mathbf{B}_{c}\right\| \\
P\left\{\gamma_{4}\left\|\widetilde{\mathbf{e}}_{c}^{T} \mathbf{P}_{1} \mathbf{B}_{c}\right\| \boldsymbol{\xi}(\widehat{\mathbf{x}})\right\}, & \left.\cdot \theta_{h_{2}}^{T} \boldsymbol{\xi}(\widehat{\mathbf{x}}(t-\tau)) \leq 0\right), \\
& \text { if }\left(\left\|\theta_{h_{2}}\right\|=M_{\theta_{h_{2}}},\right. \\
& \left\|\widetilde{\mathbf{e}}_{c}^{T} \mathbf{P}_{1} \mathbf{B}_{c}\right\| \\
& \left.\cdot \theta_{h_{2}}^{T} \boldsymbol{\xi}(\widehat{\mathbf{x}}(t-\tau))>0\right),
\end{array}\right.
\end{aligned}
$$

where $P\left\{\gamma_{4}\left\|\widetilde{\mathbf{e}}_{c}^{T} \mathbf{P}_{1} \mathbf{B}_{c}\right\| \boldsymbol{\xi}(\widehat{\mathbf{x}}(t-\tau))\right\}$ is defined as

$$
\begin{aligned}
P\left\{\gamma_{4}\right. & \left.\left\|\widetilde{\mathbf{e}}_{c}^{T} \mathbf{P}_{1} \mathbf{B}_{c}\right\| \boldsymbol{\xi}(\widehat{\mathbf{x}}(t-\tau))\right\} \\
= & \gamma_{4}\left\|\widetilde{\mathbf{e}}_{c}^{T} \mathbf{P}_{1} \mathbf{B}_{c}\right\| \boldsymbol{\xi}(\widehat{\mathbf{x}}(t-\tau)) \\
& -\gamma_{4} \frac{\theta_{h_{2}} \theta_{h_{2}}^{T}}{\left\|\theta_{h_{2}}\right\|^{2}}\left\|\widetilde{\mathbf{e}}_{c}^{T} \mathbf{P}_{1} \mathbf{B}_{c}\right\| \boldsymbol{\xi}(\widehat{\mathbf{x}}(t-\tau)) .
\end{aligned}
$$

The main result of the proposed observer-based robust adaptive fuzzy control scheme is summarized in the following theorem.

Theorem 6. Consider the MIMO nonlinear system (2) in the presence of output delay and the uncertainties subject to Assumptions 1-2. The observer-based robust adaptive fuzzy controller is defined by (19) and (44)-(45) with adaptation laws given by (46)-(49). For the given positive definite matrices $\mathbf{Q}_{1}$ and $\mathbf{Q}_{2}$, if there exist symmetric positive definite matrices $\mathbf{P}_{1}$ and $\mathbf{P}_{2}$ such that the following Lyapunov equations

$$
\begin{gathered}
\left(\mathbf{A}-\mathbf{K}_{o} \mathbf{C}\right)^{T} \mathbf{P}_{1}+\mathbf{P}_{1}\left(\mathbf{A}-\mathbf{K}_{o} \mathbf{C}\right)=-\mathbf{Q}_{1} \\
\left(\mathbf{A}-\mathbf{B K}_{c}\right)^{T} \mathbf{P}_{2}+\mathbf{P}_{2}\left(\mathbf{A}-\mathbf{B} \mathbf{K}_{c}\right)=-\mathbf{Q}_{2} \\
\mathbf{P}_{1} \mathbf{B}_{c}=\mathbf{C}^{T}
\end{gathered}
$$

are satisfied, then all the closed-loop signals are bounded, and the tracking errors converge to a neighborhood of zero.

Proof. Consider the Lyapunov function candidate

$$
\begin{aligned}
V= & \frac{1}{2} \widetilde{\mathbf{e}}_{c}^{T} \mathbf{P}_{1} \widetilde{\mathbf{e}}_{c}+\frac{1}{2} \widehat{\mathbf{e}}^{T} \mathbf{P}_{2} \widehat{\mathbf{e}}+\frac{1}{2 \gamma_{1}}\left(\widetilde{\boldsymbol{\theta}}_{1}^{T} \widetilde{\boldsymbol{\theta}}_{1}\right)+\frac{1}{2 \gamma_{2}} \operatorname{tr}\left(\widetilde{\boldsymbol{\theta}}_{2}^{T} \widetilde{\boldsymbol{\theta}}_{2}\right) \\
& +\frac{1}{2 \gamma_{3}}\left(\widetilde{\theta}_{h_{1}}^{T} \widetilde{\theta}_{h_{1}}\right)+\frac{1}{2 \gamma_{4}}\left(\widetilde{\theta}_{h_{2}}^{T} \widetilde{\theta}_{h_{2}}\right)+\frac{1}{2 \gamma_{5}} \widetilde{\omega}_{f}^{2} .
\end{aligned}
$$


By the time derivative of $V$ and the facts that $\dot{\tilde{\boldsymbol{\theta}}}_{1}=-\dot{\boldsymbol{\theta}}_{1}, \dot{\tilde{\boldsymbol{\theta}}}_{2}=$ $-\dot{\theta}_{2}, \dot{\tilde{\theta}}_{h_{1}}=-\dot{\theta}_{h_{1}}, \dot{\tilde{\theta}}_{h_{2}}=-\dot{\theta}_{h_{2}}$ and $\dot{\tilde{\omega}}_{f}=-\dot{\widehat{\omega}}_{f}$, it can be easily shown from (24) and (39) that

$$
\begin{aligned}
& \dot{V}=\frac{1}{2} \dot{\widetilde{\mathbf{e}}}_{c}^{T} \mathbf{P}_{1} \widetilde{\mathbf{e}}_{c}+\frac{1}{2} \widetilde{\mathbf{e}}_{c}^{T} \mathbf{P}_{1} \dot{\tilde{\mathbf{e}}}_{c}+\frac{1}{2} \dot{\overline{\mathbf{e}}}^{T} \mathbf{P}_{2} \widehat{\mathbf{e}}+\frac{1}{2} \widehat{\mathbf{e}}^{T} \mathbf{P}_{2} \dot{\overrightarrow{\mathbf{e}}} \\
& -\frac{1}{\gamma_{1}}\left(\widetilde{\boldsymbol{\theta}}_{1}^{T} \dot{\boldsymbol{\theta}}_{1}\right)-\frac{1}{\gamma_{2}} \operatorname{tr}\left(\widetilde{\boldsymbol{\theta}}_{2}^{T} \dot{\boldsymbol{\theta}}_{2}\right)-\frac{1}{2 \gamma_{3}}\left(\widetilde{\theta}_{h_{1}}^{T} \dot{\theta}_{h_{1}}\right) \\
& -\frac{1}{2 \gamma_{4}}\left(\widetilde{\theta}_{h_{2}}^{T} \dot{\theta}_{h_{2}}\right)-\frac{1}{\gamma_{5}} \widetilde{\omega}_{f} \dot{\vec{\omega}}_{f} \\
& =\frac{1}{2} \widetilde{\mathbf{e}}_{c}^{T}\left[\left(\mathbf{A}-\mathbf{K}_{o} \mathbf{C}\right)^{T} \mathbf{P}_{1}+\mathbf{P}_{1}\left(\mathbf{A}-\mathbf{K}_{o} \mathbf{C}\right)\right] \widetilde{\mathbf{e}}_{c} \\
& +\frac{1}{2} \widehat{\mathbf{e}}^{T}\left[\left(\mathbf{A}-\mathbf{B K}_{c}\right)^{T} \mathbf{P}_{2}+\mathbf{P}_{2}\left(\mathbf{A}-\mathbf{B} \mathbf{K}_{c}\right)\right] \widehat{\mathbf{e}} \\
& +\widetilde{\mathbf{e}}_{c}^{T} \mathbf{P}_{1} \mathbf{B}_{c}\left[\boldsymbol{\Phi}_{f}(\widehat{\mathbf{x}}) \widetilde{\boldsymbol{\theta}}_{1}+\boldsymbol{\Phi}_{f}(\widehat{\mathbf{x}}) \tilde{\boldsymbol{\theta}}_{2} \mathbf{u}(t)+\omega_{1 f}\right. \\
& \left.+\mathbf{u}_{s f}(t)+\Delta \Psi_{f}(\mathbf{x}, \mathbf{u})\right]+\widehat{\mathbf{e}}^{T} \mathbf{P}_{2} \mathbf{K}_{o} \underline{\widetilde{\mathbf{e}}_{1}} \\
& -\frac{1}{\gamma_{1}}\left(\widetilde{\boldsymbol{\theta}}_{1}^{T} \dot{\boldsymbol{\theta}}_{1}\right)-\frac{1}{\gamma_{2}} \operatorname{tr}\left(\widetilde{\boldsymbol{\theta}}_{2}^{T} \dot{\boldsymbol{\theta}}_{2}\right)-\frac{1}{2 \gamma_{3}}\left(\widetilde{\theta}_{h_{1}}^{T} \dot{\theta}_{h_{1}}\right) \\
& -\frac{1}{2 \gamma_{4}}\left(\widetilde{\theta}_{h_{2}}^{T} \dot{\theta}_{h_{2}}\right)-\frac{1}{\gamma_{5}} \widetilde{\omega}_{f} \dot{\hat{\omega}}_{f} \\
& \leq \frac{1}{2} \widetilde{\mathbf{e}}_{c}^{T}\left[\left(\mathbf{A}-\mathbf{K}_{o} \mathbf{C}\right)^{T} \mathbf{P}_{1}+\mathbf{P}_{1}\left(\mathbf{A}-\mathbf{K}_{o} \mathbf{C}\right)\right] \widetilde{\mathbf{e}}_{c} \\
& +\frac{1}{2} \widehat{\mathbf{e}}^{T}\left[\left(\mathbf{A}-\mathbf{B K}_{c}\right)^{T} \mathbf{P}_{2}+\mathbf{P}_{2}\left(\mathbf{A}-\mathbf{B} \mathbf{K}_{c}\right)\right] \widehat{\mathbf{e}} \\
& +\widetilde{\mathbf{e}}_{c}^{T} \mathbf{P}_{1} \mathbf{B}_{c} \boldsymbol{\Phi}_{f}(\widehat{\mathbf{x}}) \widetilde{\boldsymbol{\theta}}_{1}+\widetilde{\mathbf{e}}_{c}^{T} \mathbf{P}_{1} \mathbf{B}_{c} \boldsymbol{\Phi}_{f}(\widehat{\mathbf{x}}) \widetilde{\boldsymbol{\theta}}_{2} \mathbf{u} \\
& +\widetilde{\mathbf{e}}_{c}^{T} \mathbf{P}_{1} \mathbf{B}_{c} \mathbf{u}_{s f}+\left\|\widetilde{\mathbf{e}}_{c}^{T} \mathbf{P}_{1} \mathbf{B}_{c}\right\|\left\|\boldsymbol{\omega}_{1 f}\right\| \\
& +\left\|\widetilde{\mathbf{e}}_{c}^{T} \mathbf{P}_{1} \mathbf{B}_{c}\right\| \cdot\left\|\Delta \Psi_{f}(\mathbf{x}, \mathbf{u})\right\|+\widehat{\mathbf{e}}^{T} \mathbf{P}_{2} \mathbf{K}_{o} \underline{\widetilde{\mathbf{e}}_{1}} \\
& -\frac{1}{\gamma_{1}}\left(\widetilde{\boldsymbol{\theta}}_{1}^{T} \dot{\boldsymbol{\theta}}_{1}\right)-\frac{1}{\gamma_{2}} \operatorname{tr}\left(\widetilde{\boldsymbol{\theta}}_{2}^{T} \dot{\boldsymbol{\theta}}_{2}\right)-\frac{1}{2 \gamma_{3}}\left(\widetilde{\theta}_{h_{1}}^{T} \dot{\theta}_{h_{1}}\right) \\
& -\frac{1}{2 \gamma_{4}}\left(\widetilde{\theta}_{h_{2}}^{T} \dot{\theta}_{h_{2}}\right)-\frac{1}{\gamma_{5}} \widetilde{\omega}_{f} \dot{\vec{\omega}}_{f} \text {. }
\end{aligned}
$$

Applying Assumption 2, (56), and (57), it yields

$$
\begin{aligned}
\dot{V} \leq & -\frac{1}{2} \widetilde{\mathbf{e}}_{c}^{T} \mathbf{Q}_{1} \widetilde{\mathbf{e}}_{c}-\frac{1}{2} \widehat{\mathbf{e}}^{T} \mathbf{Q}_{2} \widehat{\mathbf{e}}+\widetilde{\mathbf{e}}_{c}^{T} \mathbf{P}_{1} \mathbf{B}_{c} \boldsymbol{\Phi}_{f}(\widehat{\mathbf{x}}) \widetilde{\boldsymbol{\theta}}_{1} \\
& +\widetilde{\mathbf{e}}_{c}^{T} \mathbf{P}_{1} \mathbf{B}_{c} \boldsymbol{\Phi}_{f}(\widehat{\mathbf{x}}) \widetilde{\boldsymbol{\theta}}_{2} \mathbf{u}+\widetilde{\mathbf{e}}_{c}^{T} \mathbf{P}_{1} \mathbf{B}_{c} \mathbf{u}_{s f}+\left\|\widetilde{\mathbf{e}}_{c}^{T} \mathbf{P}_{1} \mathbf{B}_{c}\right\|\left\|\boldsymbol{\omega}_{1 f}\right\| \\
& +\left\|\widetilde{\mathbf{e}}_{c}^{T} \mathbf{P}_{1} \mathbf{B}_{c}\right\| \cdot\left(h_{1}(\mathbf{x})+h_{2}(\mathbf{x}(t-\tau))\right)+\widehat{\mathbf{e}}^{T} \mathbf{P}_{2} \mathbf{K}_{o} \widetilde{\mathbf{e}}_{1} \\
& -\frac{1}{\gamma_{1}}\left(\widetilde{\boldsymbol{\theta}}_{1}^{T} \dot{\boldsymbol{\theta}}_{1}\right)-\frac{1}{\gamma_{2}} \operatorname{tr}\left(\widetilde{\boldsymbol{\theta}}_{2}^{T} \dot{\boldsymbol{\theta}}_{2}\right)-\frac{1}{2 \gamma_{3}}\left(\widetilde{\theta}_{h_{1}}^{T} \dot{\theta}_{h_{1}}\right) \\
& -\frac{1}{2 \gamma_{4}}\left(\widetilde{\theta}_{h_{2}}^{T} \dot{\theta}_{h_{2}}\right)-\frac{1}{\gamma_{5}} \widetilde{\omega}_{f} \dot{\hat{\omega}}_{f}
\end{aligned}
$$

$$
\begin{gathered}
=-\frac{1}{2} \widetilde{\mathbf{e}}_{c}^{T} \mathbf{Q}_{1} \widetilde{\mathbf{e}}_{c}-\frac{1}{2} \widehat{\mathbf{e}}^{T} \mathbf{Q}_{2} \widehat{\mathbf{e}}+\widetilde{\mathbf{e}}_{c}^{T} \mathbf{P}_{1} \mathbf{B}_{c} \boldsymbol{\Phi}_{f}(\widehat{\mathbf{x}}) \widetilde{\boldsymbol{\theta}}_{1} \\
+\widetilde{\mathbf{e}}_{c}^{T} \mathbf{P}_{1} \mathbf{B}_{c} \boldsymbol{\Phi}_{f}(\widehat{\mathbf{x}}) \widetilde{\boldsymbol{\theta}}_{2} \mathbf{u}+\widetilde{\mathbf{e}}_{c}^{T} \mathbf{P}_{1} \mathbf{B}_{c} \mathbf{u}_{s f}+\left\|\widetilde{\mathbf{e}}_{c}^{T} \mathbf{P}_{1} \mathbf{B}_{c}\right\|\left\|\boldsymbol{\omega}_{1 f}\right\| \\
+\left\|\widetilde{\mathbf{e}}_{c}^{T} \mathbf{P}_{1} \mathbf{B}_{c}\right\| \cdot\left[h_{1}(\mathbf{x})-\widehat{h}_{1}\left(\widehat{\mathbf{x}} \mid \theta_{h_{1}}^{*}\right)+\widehat{h}_{1}\left(\widehat{\mathbf{x}} \mid \theta_{h_{1}}^{*}\right)\right. \\
\left.-\widehat{h}_{1}\left(\widehat{\mathbf{x}} \mid \theta_{h_{1}}\right)+\widehat{h}_{1}\left(\widehat{\mathbf{x}} \mid \theta_{h_{1}}\right)\right] \\
+\left\|\widetilde{\mathbf{e}}_{c}^{T} \mathbf{P}_{1} \mathbf{B}_{c}\right\| \cdot\left[h_{2}(\mathbf{x}(t-\tau))-\widehat{h}_{2}\left(\widehat{\mathbf{x}}(t-\tau) \mid \theta_{h_{2}}^{*}\right)\right. \\
+\widehat{h}_{2}\left(\widehat{\mathbf{x}}(t-\tau) \mid \theta_{h_{2}}^{*}\right) \\
\quad-\widehat{h}_{2}\left(\widehat{\mathbf{x}}(t-\tau) \mid \theta_{h_{2}}\right) \\
\left.+\widehat{h}_{2}\left((t-\tau) \widehat{\mathbf{x}} \mid \theta_{h_{2}}\right)\right] \\
+\widehat{\mathbf{e}}^{T} \mathbf{P}_{2} \mathbf{K}_{o} \widetilde{\mathbf{e}}_{1}-\frac{1}{\gamma_{1}}\left(\widetilde{\boldsymbol{\theta}}_{1}^{T} \dot{\boldsymbol{\theta}}_{1}\right)-\frac{1}{\gamma_{2}} \operatorname{tr}\left(\widetilde{\boldsymbol{\theta}}_{2}^{T} \dot{\boldsymbol{\theta}}_{2}\right) \\
-\frac{1}{2 \gamma_{3}}\left(\widetilde{\theta}_{h_{1}}^{T} \dot{\theta}_{h_{1}}\right)-\frac{1}{2 \gamma_{4}}\left(\widetilde{\theta}_{h_{2}}^{T} \dot{\theta}_{h_{2}}\right)-\frac{1}{\gamma_{5}} \widetilde{\boldsymbol{\omega}}_{f} \dot{\hat{\boldsymbol{\omega}}}_{f} .
\end{gathered}
$$

According to (33) and (43), we have

$$
\begin{aligned}
& \dot{V} \leq-\frac{1}{2} \widetilde{\mathbf{e}}_{c}^{T} \mathbf{Q}_{1} \widetilde{\mathbf{e}}_{c}-\frac{1}{2} \widehat{\mathbf{e}}^{T} \mathbf{Q}_{2} \widehat{\mathbf{e}}+\widetilde{\mathbf{e}}_{c}^{T} \mathbf{P}_{1} \mathbf{B}_{c} \boldsymbol{\Phi}_{f}(\widehat{\mathbf{x}}) \widetilde{\boldsymbol{\theta}}_{1} \\
& +\widetilde{\mathbf{e}}_{c}^{T} \mathbf{P}_{1} \mathbf{B}_{c} \boldsymbol{\Phi}_{f}(\widehat{\mathbf{x}}) \tilde{\boldsymbol{\theta}}_{2} \mathbf{u}+\widetilde{\mathbf{e}}_{c}^{T} \mathbf{P}_{1} \mathbf{B}_{c} \mathbf{u}_{s f}+\left\|\widetilde{\mathbf{e}}_{c}^{T} \mathbf{P}_{1} \mathbf{B}_{c}\right\|\left\|\boldsymbol{\omega}_{1 f}\right\| \\
& +\left\|\widetilde{\mathbf{e}}_{c}^{T} \mathbf{P}_{1} \mathbf{B}_{c}\right\| \cdot \widehat{h}_{1}\left(\widehat{\mathbf{x}} \mid \theta_{h_{1}}\right)+\left\|\widetilde{\mathbf{e}}_{c}^{T} \mathbf{P}_{1} \mathbf{B}_{c}\right\| \cdot \widetilde{\theta}_{h_{1}}^{T} \xi(\widehat{\mathbf{x}}) \\
& +\left\|\widetilde{\mathbf{e}}_{c}^{T} \mathbf{P}_{1} \mathbf{B}_{c}\right\| \cdot \widehat{h}_{2}\left(\widehat{\mathbf{x}}(t-\tau) \mid \theta_{h_{2}}\right) \\
& +\left\|\tilde{\mathbf{e}}_{c}^{T} \mathbf{P}_{1} \mathbf{B}_{c}\right\| \cdot \tilde{\theta}_{h_{2}}^{T} \xi(\widehat{\mathbf{x}}(t-\tau)) \\
& +\left\|\widetilde{\mathbf{e}}_{c}^{T} \mathbf{P}_{1} \mathbf{B}_{c}\right\| \cdot\left|\omega_{2}\right|+\widehat{\mathbf{e}}^{T} \mathbf{P}_{2} \mathbf{K}_{o} \underline{\widetilde{\mathbf{e}}_{1}}-\frac{1}{\gamma_{1}}\left(\widetilde{\boldsymbol{\theta}}_{1}^{T} \dot{\boldsymbol{\theta}}_{1}\right) \\
& -\frac{1}{\gamma_{2}} \operatorname{tr}\left(\widetilde{\boldsymbol{\theta}}_{2}^{T} \dot{\boldsymbol{\theta}}_{2}\right)-\frac{1}{2 \gamma_{3}}\left(\widetilde{\theta}_{h_{1}}^{T} \dot{\theta}_{h_{1}}\right)-\frac{1}{2 \gamma_{4}}\left(\widetilde{\theta}_{h_{2}}^{T} \dot{\theta}_{h_{2}}\right) \\
& -\frac{1}{\gamma_{5}} \widetilde{\omega}_{f} \dot{\widehat{\omega}}_{f} \\
& \leq-\frac{1}{2} \widetilde{\mathbf{e}}_{c}^{T} \mathbf{Q}_{1} \widetilde{\mathbf{e}}_{c}-\frac{1}{2} \widehat{\mathbf{e}}^{T} \mathbf{Q}_{2} \widehat{\mathbf{e}}+\widetilde{\mathbf{e}}_{c}^{T} \mathbf{P}_{1} \mathbf{B}_{c} \boldsymbol{\Phi}_{f}(\widehat{\mathbf{x}}) \widetilde{\boldsymbol{\theta}}_{1} \\
& +\widetilde{\mathbf{e}}_{c}^{T} \mathbf{P}_{1} \mathbf{B}_{c} \boldsymbol{\Phi}_{f}(\widehat{\mathbf{x}}) \widetilde{\boldsymbol{\theta}}_{2} \mathbf{u}+\widetilde{\mathbf{e}}_{c}^{T} \mathbf{P}_{1} \mathbf{B}_{c} \mathbf{u}_{s f}+\left\|\widetilde{\mathbf{e}}_{c}^{T} \mathbf{P}_{1} \mathbf{B}_{c}\right\| \widehat{\omega}_{f} \\
& +\left\|\widetilde{\mathbf{e}}_{c}^{T} \mathbf{P}_{1} \mathbf{B}_{c}\right\| \cdot \widehat{h}_{1}\left(\widehat{\mathbf{x}} \mid \theta_{h_{1}}\right)+\left\|\tilde{\mathbf{e}}_{c}^{T} \mathbf{P}_{1} \mathbf{B}_{c}\right\| \cdot \tilde{\theta}_{h_{1}}^{T} \xi(\widehat{\mathbf{x}}) \\
& +\left\|\widetilde{\mathbf{e}}_{c}^{T} \mathbf{P}_{1} \mathbf{B}_{c}\right\| \cdot \widehat{h}_{2}\left(\widehat{\mathbf{x}}(t-\tau) \mid \theta_{h_{2}}\right) \\
& +\left\|\widetilde{\mathbf{e}}_{c}^{T} \mathbf{P}_{1} \mathbf{B}_{c}\right\| \cdot \widetilde{\theta}_{h_{2}}^{T} \xi(\widehat{\mathbf{x}}(t-\tau))
\end{aligned}
$$




$$
\begin{aligned}
& +\left\|\widetilde{\mathbf{e}}_{c}^{T} \mathbf{P}_{1} \mathbf{B}_{c}\right\| \cdot \widetilde{\omega}_{f}+\widehat{\hat{\mathbf{e}}}^{T} \mathbf{P}_{2} \mathbf{K}_{o} \underline{\tilde{\mathbf{e}}_{1}}-\frac{1}{\gamma_{1}}\left(\widetilde{\boldsymbol{\theta}}_{1}^{T} \dot{\boldsymbol{\theta}}_{1}\right) \\
& -\frac{1}{\gamma_{2}} \operatorname{tr}\left(\widetilde{\boldsymbol{\theta}}_{2}^{T} \dot{\boldsymbol{\theta}}_{2}\right)-\frac{1}{2 \gamma_{3}}\left(\widetilde{\theta}_{h_{1}}^{T} \dot{\theta}_{h_{1}}\right) \\
& -\frac{1}{2 \gamma_{4}}\left(\widetilde{\theta}_{h_{2}}^{T} \dot{\theta}_{h_{2}}\right)-\frac{1}{\gamma_{5}} \widetilde{\omega}_{f} \dot{\widehat{\omega}}_{f} \\
& =-\frac{1}{2} \widetilde{\mathbf{e}}_{c}^{T} \mathbf{Q}_{1} \widetilde{\mathbf{e}}_{c}-\frac{1}{2} \widehat{\mathbf{e}}^{T} \mathbf{Q}_{2} \widehat{\mathbf{e}}+\widetilde{\mathbf{e}}_{c}^{T} \mathbf{P}_{1} \mathbf{B}_{c} \mathbf{u}_{s f} \\
& +\left\|\widetilde{\mathbf{e}}_{c}^{T} \mathbf{P}_{1} \mathbf{B}_{c}\right\| \widehat{\omega}_{f}+\left\|\widetilde{\mathbf{e}}_{c}^{T} \mathbf{P}_{1} \mathbf{B}_{c}\right\| \cdot \widehat{h}_{1}\left(\widehat{\mathbf{x}} \mid \theta_{h_{1}}\right) \\
& +\left\|\widetilde{\boldsymbol{e}}_{c}^{T} \mathbf{P}_{1} \mathbf{B}_{c}\right\| \cdot \widehat{h}_{2}\left(\widehat{\mathbf{x}}(t-\tau) \mid \theta_{h_{2}}\right)+\widehat{\mathbf{e}}^{T} \mathbf{P}_{2} \mathbf{K}_{o} \underline{\tilde{\mathbf{e}}_{1}} \\
& +\left[\widetilde{\mathbf{e}}_{c}^{T} \mathbf{P}_{1} \mathbf{B}_{c} \boldsymbol{\Phi}_{f}(\widehat{\mathbf{x}}) \widetilde{\boldsymbol{\theta}}_{1}-\frac{1}{\gamma_{1}}\left(\widetilde{\boldsymbol{\theta}}_{1}^{T} \dot{\boldsymbol{\theta}}_{1}\right)\right] \\
& +\left[\widetilde{\mathbf{e}}_{c}^{T} \mathbf{P}_{1} \mathbf{B}_{c} \boldsymbol{\Phi}_{f}(\widehat{\mathbf{x}}) \widetilde{\boldsymbol{\theta}}_{2} \mathbf{u}-\frac{1}{\gamma_{2}} \operatorname{tr}\left(\widetilde{\boldsymbol{\theta}}_{2}^{T} \dot{\boldsymbol{\theta}}_{2}\right)\right] \\
& +\left[\left\|\tilde{\mathbf{e}}_{c}^{T} \mathbf{P}_{1} \mathbf{B}_{c}\right\| \cdot \tilde{\theta}_{h_{1}}^{T} \xi(\widehat{\mathbf{x}})-\frac{1}{\gamma_{3}}\left(\widetilde{\theta}_{h_{1}}^{T} \dot{\theta}_{h_{1}}\right)\right] \\
& +\left[\left\|\tilde{\mathbf{e}}_{c}^{T} \mathbf{P}_{1} \mathbf{B}_{c}\right\| \cdot \widetilde{\theta}_{h_{2}}^{T} \xi(\widehat{\mathbf{x}}(t-\tau))-\frac{1}{\gamma_{4}}\left(\widetilde{\theta}_{h_{2}}^{T} \dot{\theta}_{h_{2}}\right)\right] \\
& +\left[\left\|\widetilde{\mathbf{e}}_{c}^{T} \mathbf{P}_{1} \mathbf{B}_{c}\right\| \widetilde{\omega}_{f}-\frac{1}{\gamma_{5}} \widetilde{\omega}_{f} \dot{\widehat{\omega}}_{f}\right] .
\end{aligned}
$$

By employing (46)-(49) and using the control laws (44)-(45), we can obtain

$$
\dot{V} \leq-\frac{1}{2} \widetilde{\mathbf{e}}_{c}^{T} \mathbf{Q}_{1} \widetilde{\mathbf{e}}_{c}-\frac{1}{2} \widehat{\mathbf{e}}^{T} \mathbf{Q}_{2} \widehat{\mathbf{e}} .
$$

Therefore, it can be concluded that $\dot{V} \leq 0$ from (63), and the output tracking error of the closed-loop system converges asymptotically to a neighborhood of zero based on the Lyapunov synthesis approach. This completes the proof.

\section{An Example and Simulation Results}

In this paper, a numerical example is illustrated to verify the performance of the proposed observer-based robust adaptive fuzzy controller. Consider the following MIMO nonlinear uncertain system with delayed output:

$$
\begin{gathered}
\dot{x}_{11}(t)=x_{12}(t), \\
\dot{x}_{12}(t)=f_{1}(\mathbf{x})+g_{11}(\mathbf{x}) u_{1}(t)+g_{12}(\mathbf{x}) u_{2}(t)+\Delta \phi_{1}(\mathbf{x}), \\
\dot{x}_{21}(t)=x_{22}(t), \\
\dot{x}_{22}(t)=f_{2}(\mathbf{x})+g_{21}(\mathbf{x}) u_{1}(t)+g_{22}(\mathbf{x}) u_{2}(t)+\Delta \phi_{2}(\mathbf{x}), \\
y_{1}(t)=x_{11}(t-\tau), \\
y_{2}(t)=x_{21}(t-\tau) .
\end{gathered}
$$

According to (2), the above equation can be rewritten as the following compact form:

$$
\begin{aligned}
& {\left[\begin{array}{l}
\dot{x}_{11}(t) \\
\dot{x}_{12}(t) \\
\dot{x}_{21}(t) \\
\dot{x}_{22}(t)
\end{array}\right]=\left[\begin{array}{llll}
0 & 1 & 0 & 0 \\
0 & 0 & 0 & 0 \\
0 & 0 & 0 & 1 \\
0 & 0 & 0 & 0
\end{array}\right]\left[\begin{array}{l}
x_{11}(t) \\
x_{12}(t) \\
x_{21}(t) \\
x_{22}(t)
\end{array}\right]+\left[\begin{array}{ll}
0 & 0 \\
1 & 0 \\
0 & 0 \\
0 & 1
\end{array}\right]} \\
& \times\left(\left[\begin{array}{l}
f_{1}(\mathbf{x}) \\
f_{2}(\mathbf{x})
\end{array}\right]+\left[\begin{array}{ll}
g_{11}(\mathbf{x}) & g_{12}(\mathbf{x}) \\
g_{21}(\mathbf{x}) & g_{22}(\mathbf{x})
\end{array}\right]\left[\begin{array}{l}
u_{1}(t) \\
u_{2}(t)
\end{array}\right]\right. \\
& \left.+\left[\begin{array}{c}
\Delta \phi_{1}(\mathbf{x}) \\
\Delta \phi_{2}(\mathbf{x})
\end{array}\right]\right) \\
& {\left[\begin{array}{l}
y_{1}(t) \\
y_{2}(t)
\end{array}\right]=\left[\begin{array}{llll}
1 & 0 & 0 & 0 \\
0 & 0 & 1 & 0
\end{array}\right]\left[\begin{array}{l}
x_{11}(t-\tau) \\
x_{12}(t-\tau) \\
x_{21}(t-\tau) \\
x_{22}(t-\tau)
\end{array}\right] \text {, }}
\end{aligned}
$$

where $\mathbf{A}_{1}=\mathbf{A}_{2}=\left[\begin{array}{ll}0 & 1 \\ 0 & 0\end{array}\right], \mathbf{B}_{1}=\mathbf{B}_{2}=\left[\begin{array}{l}0 \\ 1\end{array}\right], \mathbf{C}_{1}=\mathbf{C}_{2}=\left[\begin{array}{ll}1 & 0\end{array}\right]$, the nonlinear system functions $f_{1}(\mathbf{x})=\left(1-e^{-x_{11}}\right) /\left(1+e^{-x_{11}}\right)$, $f_{2}(\mathbf{x})=\left(1-e^{-x_{21}}\right) /\left(1+e^{-x_{21}}\right), g_{12}(\mathbf{x})=g_{21}(\mathbf{x})=0$, and $g_{11}(\mathbf{x})=g_{22}(\mathbf{x})=1$, and the uncertainties $\Delta \boldsymbol{\Phi}=\left[\begin{array}{l}0.1 x_{21} \sin (t) \\ 0.1 x_{12} \cos (t)\end{array}\right]$ and the delay time is $\tau=0.1$. In the simulation, these nonlinear system functions and uncertainties are assumed to be unknown and are estimated by the fuzzy logic systems. The fuzzy membership functions are chosen as follows:

$$
\begin{aligned}
& \mu_{F_{i}^{1}}\left(\widehat{x}_{i}\right)=\frac{1}{1+\exp \left(5\left(\hat{x}_{i}+0.8\right)\right)}, \\
& \mu_{F_{i}^{2}}\left(\widehat{x}_{i}\right)=\exp \left(-\left(\widehat{x}_{i}+0.6\right)^{2}\right), \\
& \mu_{F_{i}^{3}}\left(\widehat{x}_{i}\right)=\exp \left(-\left(\widehat{x}_{i}+0.4\right)^{2}\right), \\
& \mu_{F_{i}^{4}}\left(\widehat{x}_{i}\right)=\exp \left(-\widehat{x}_{i}^{2}\right), \\
& \mu_{F_{i}^{5}}\left(\widehat{x}_{i}\right)=\exp \left(-\left(\widehat{x}_{i}-0.4\right)^{2}\right), \\
& \mu_{F_{i}^{6}}\left(\widehat{x}_{i}\right)=\exp \left(-\left(\hat{x}_{i}-0.6\right)^{2}\right), \\
& \mu_{F_{i}^{7}}\left(\widehat{x}_{i}\right)=\frac{1}{1+\exp \left(-5\left(\widehat{x}_{i}-0.8\right)\right)}, \quad i=1,2,3,4 .
\end{aligned}
$$

Let

$$
\begin{gathered}
\xi^{l}(\widehat{x})=\frac{\mu_{F_{1}^{l}}\left(\widehat{x}_{1}\right) \mu_{F_{2}^{l}}\left(\widehat{x}_{2}\right) \mu_{F_{3}^{l}}\left(\widehat{x}_{3}\right) \mu_{F_{4}^{l}}\left(\widehat{x}_{4}\right)}{\sum_{l=1}^{7} \mu_{F_{1}^{l}}\left(\widehat{x}_{1}\right) \mu_{F_{2}^{l}}\left(\widehat{x}_{2}\right) \mu_{F_{3}^{l}}\left(\widehat{x}_{3}\right) \mu_{F_{4}^{l}}\left(\widehat{x}_{4}\right)}, \\
\xi(\widehat{x})=\left(\xi^{1}(\widehat{x}), \xi^{2}(\widehat{x}), \xi^{3}(\widehat{x}), \xi^{4}(\widehat{x}), \xi^{5}(\widehat{x}), \xi^{6}(\widehat{x}), \xi^{7}(\widehat{x})\right)^{T} .
\end{gathered}
$$

In this section, we apply the observer-based robust adaptive fuzzy controller proposed in Section 3 to deal with the output tracking control problem of an MIMO nonlinear uncertain system with delayed output in (65).

The control objective is to force the system output to track the given desired trajectories $y_{1 d}=y_{2 d}=0.5 \sin (2 \pi / 10)$. 


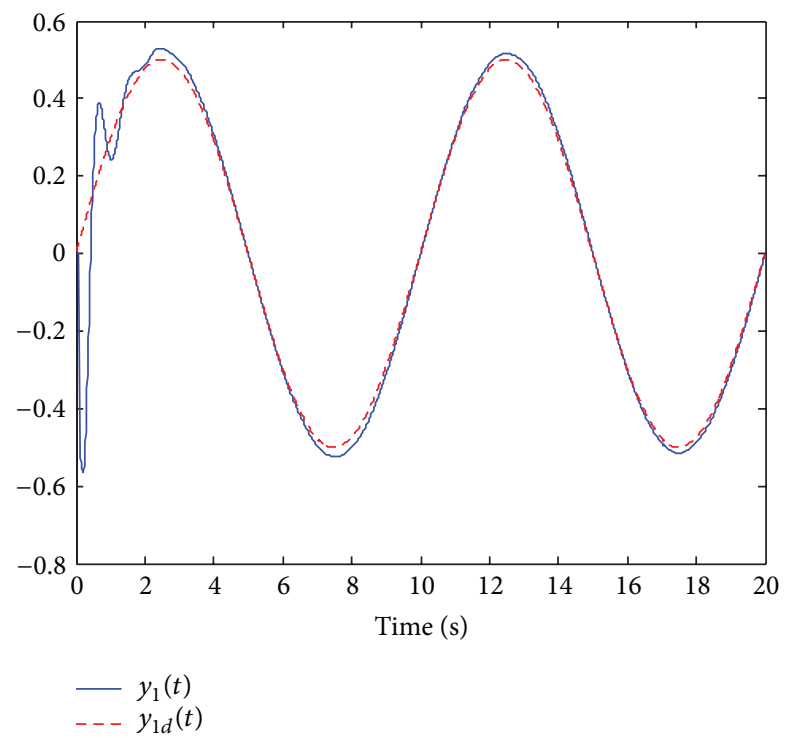

FIgURE 1: The trajectories of $y_{1}$ and $y_{1 d}$.

First, we select the control and observer gain matrices as $\mathbf{K}_{c}=$ $\left[\begin{array}{cccc}170 & 40 & 0 & 0 \\ 0 & 0 & 180 & 30\end{array}\right]$ and $\mathbf{K}_{o}=\left[\begin{array}{cccc}50 & 100 & 0 & 0 \\ 0 & 0 & 75 & 100\end{array}\right]^{T}$, using (20) and (21) to approximate the unknown $\mathbf{F}(\mathbf{x})$ and $\mathbf{G}(\mathbf{x})$, respectively. Given the positive definite matrices $\mathbf{Q}_{1}=\operatorname{diag}[1,1,1,1]$ and $\mathbf{Q}_{2}=$ $\operatorname{diag}[1,1,1,1]$, and solving the Lyapunov equations (56), we can get

$$
\begin{aligned}
& \mathbf{P}_{1}=\left[\begin{array}{cccc}
0.0101 & 0.005 & 0 & 0 \\
0.005 & 1.26 & 0 & 0 \\
0 & 0 & 0.0067 & 0.005 \\
0 & 0 & 0.005 & 1.0483
\end{array}\right], \\
& \mathbf{P}_{2}=\left[\begin{array}{cccc}
0.1302 & -0.5 & 0 & 0 \\
-0.5 & 2.1375 & 0 & 0 \\
0 & 0 & 0.1 & -0.5 \\
0 & 0 & -0.5 & 3.0167
\end{array}\right] .
\end{aligned}
$$

The initial conditions are chosen as $\mathbf{x}(0)=$ $[-0.5,-0.5,0.5,0.5]^{T}, \widehat{\mathbf{e}}(0)=[0.3,0.3,0.3,0.3]^{T}, \boldsymbol{\theta}_{h}(0)=\mathbf{0}$, $\theta_{f 1}(0)=\theta_{f 2}(0)=0, \gamma_{1}=\gamma_{2}=0.13, \gamma_{3}=0.1, \gamma_{4}=0.01$, $\theta_{g 11}(0)=1, \theta_{g 12}(0)=0, \theta_{g 21}(0)=0, \theta_{g 22}(0)=1$. The Simulation results are shown in Figures 1, 2, 3, 4, 5, 6, and 7. Figures 1 and 2 show the output tracking performance of the system output. Figures 3, 4, 5, and 6 show the trajectories of the system states and their estimation states. Figure 7 shows the control signal. Obviously, the proposed controller can not only ensure that all the signals of the resulting closed-loop system are bounded but also obtain that the good tracking performance of an MIMO nonlinear uncertain output-delay system is achieved.

\section{Conclusion}

For a class of multiinput multioutput (MIMO) nonlinear systems with output delay and uncertainties, a robust adaptive fuzzy output feedback controller is proposed to deal with

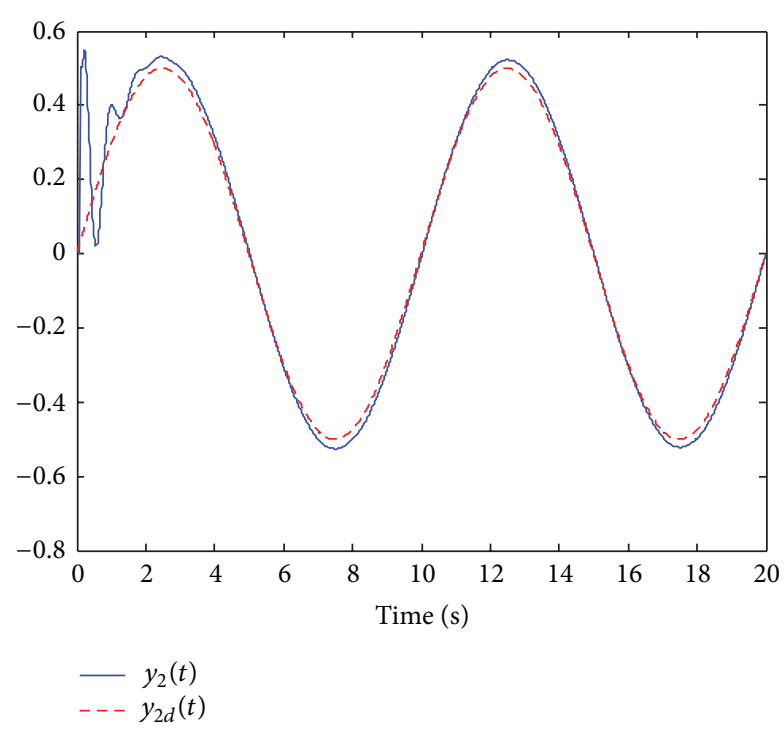

Figure 2: The trajectories of $y_{2}$ and $y_{2 d}$.

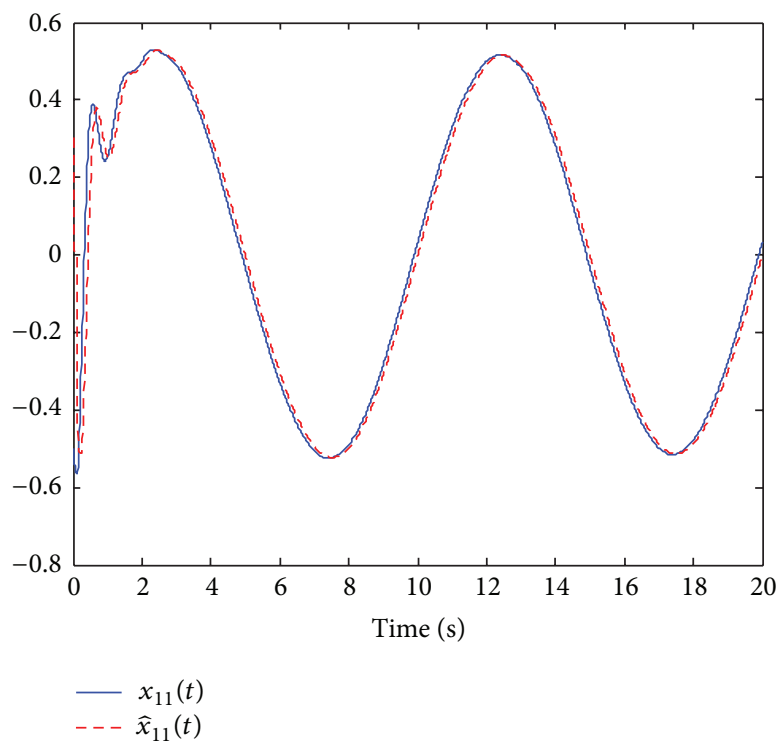

FIGURE 3: The trajectories of $x_{11}$ and $\widehat{x}_{11}$.

the problem of robust stability and output tracking control. Since the system states are unavailable for measurement, the elements of the observation error vector cannot be obtained completely. Hence, by means of the strictly positive-real (SPR) theory, all elements of the observation error vector will be obtained completely. Moreover, the fuzzy logic systems and some parameter adaptive laws are used to approximate the unknown nonlinear functions and the unknown upper bounds of the uncertainties including the structural uncertainty. By constructing an appropriate Lyapunov function, the proposed observer-based robust adaptive fuzzy controller can not only guarantee that all the signals in the whole closed-loop system are bounded but also obtain that the 


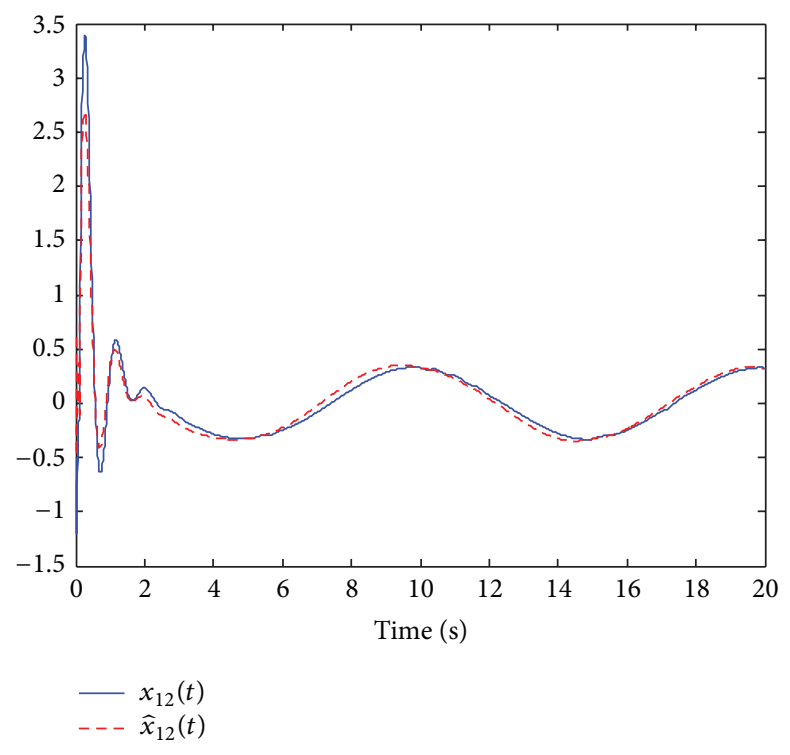

FIgURE 4: The trajectories of $x_{12}$ and $\hat{x}_{12}$.

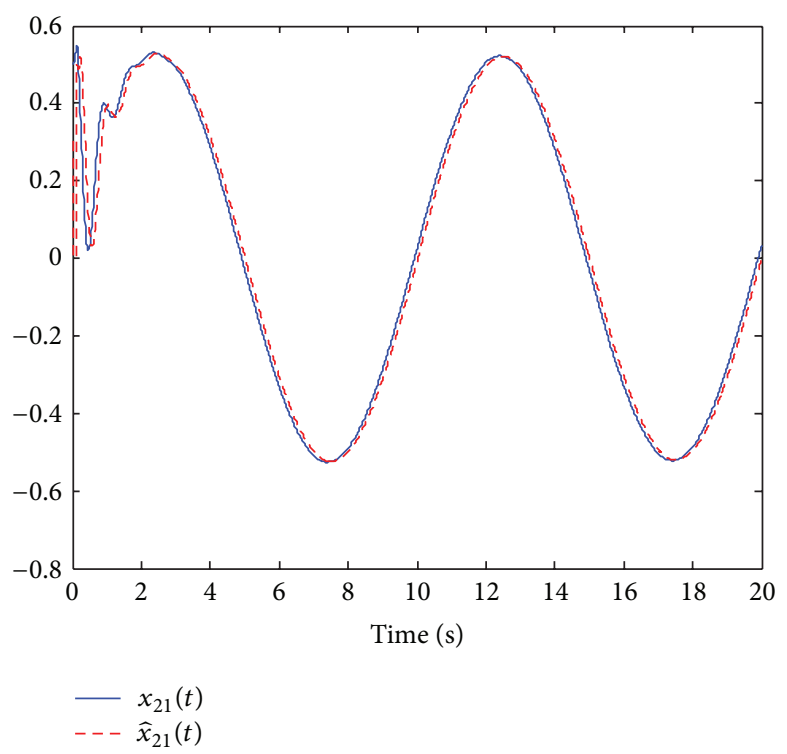

FIGURE 5: The trajectories of $x_{21}$ and $\hat{x}_{21}$.

output tracking performance of the controlled system with delayed output and uncertainties can be achieved. Finally, some simulation results are provided to illustrate the validity of the proposed approach.

\section{References}

[1] A. Isidori, Nonlinear Control Systems, Springer, New York, NY, USA, 2nd edition, 1989.

[2] J. E. Slotine and W. Li, Applied Nonlinear Control, Prentice-Hall, Englewood Cliffs, NJ, USA, 1991.

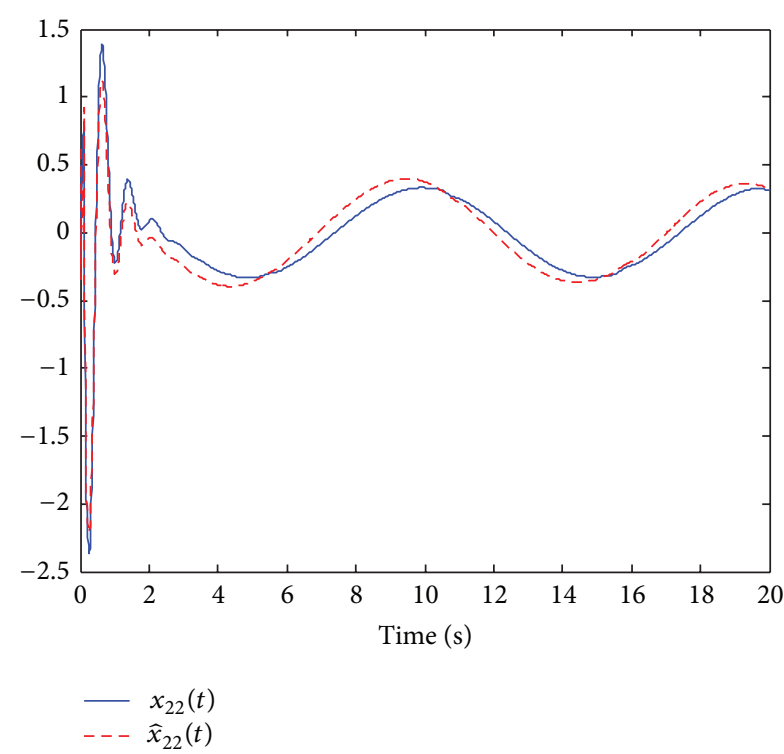

Figure 6: The trajectories of $x_{22}$ and $\hat{x}_{22}$.

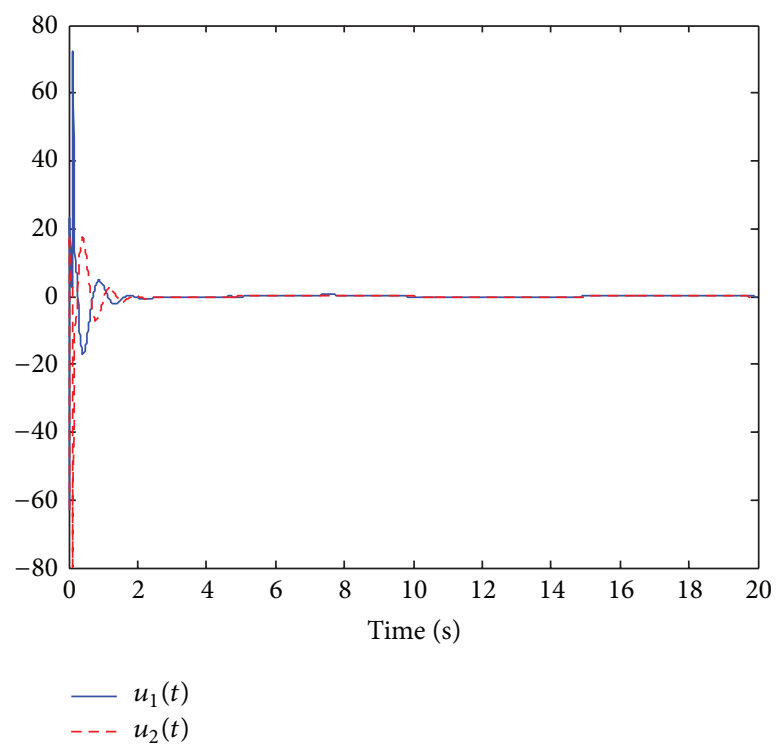

FIGURE 7: The control signals $u_{1}$ and $u_{2}$.

[3] C. P. Huang, "Model based fuzzy control with affine T-S delayed models applied to nonlinear systems," International Journal of Innovative Computing Information and Control, vol. 8, no. 5, pp. 2979-2993, 2012.

[4] Y. Yang, "Direct robust adaptive fuzzy control (DRAFC) for uncertain nonlinear systems using small gain theorem," Fuzzy Sets and Systems, vol. 151, no. 1, pp. 79-97, 2005.

[5] C. C. Chiang and Z. H. Kuo, "Output tracking controller design for MIMO nonlinear systems with higher-order and unmatched uncertainties," in Proceedings of the 39th IEEE Confernce on Decision and Control, pp. 1315-1320, December 2000.

[6] Y. Wang, Y. Feng, X. Yu, and N. Zhang, "Terminal sliding mode control of MIMO linear systems with unmatched uncertainties," 
in Proceedings of the 29th Annual Conference of the IEEE Industrial Electronics Society, pp. 1146-1151, November 2003.

[7] C. C. Chiang and T. C. Tung, "Robust tracking control of uncertain nonlinear systems with an input time elay," in Proceedings of the International Joint Conference on Neural Networks, pp. 23942399, July 2003.

[8] C. Qian and W. Lin, "Output feedback control of a class of nonlinear systems: a nonseparation principle paradigm," IEEE Transactions on Automatic Control, vol. 47, no. 10, pp. 1710-1715, 2002.

[9] L. Wu and W. X. Zheng, " $\mathrm{L}_{2}-\mathrm{L}_{\infty}$ control of nonlinear fuzzy itô stochastic delay systems via dynamic output feedback," IEEE Transactions on Systems, Man, and Cybernetics B, vol. 39, no. 5, pp. 1308-1315, 2009.

[10] C. S. Chiu, "Robust adaptive control of uncertain MIMO nonlinear system-feedforward Takagi-Sugeno fuzzy approximation based approach," IEE Proceedings Control Theory and Applications, vol. 152, no. 2, pp. 157-164, 2005.

[11] S. Labiod, M. S. Boucherit, and T. M. Guerra, "Adaptive fuzzy control of a class of MIMO nonlinear systems," Fuzzy Sets and Systems, vol. 151, no. 1, pp. 59-77, 2005.

[12] L. X. Wang, Adaptive Fuzzy Systems and Control: Design and Stability Analysis, Prentice-Hill, Englewood Cliffs, NJ, USA, 1994.

[13] B. S. Chen, C. H. Lee, and Y. C. Chang, " $H_{\infty}$ tracking design of uncertain nonlinear SISO systems: adaptive fuzzy approach," IEEE Transactions on Fuzzy Systems, vol. 4, no. 1, pp. 32-43, 1996.

[14] S. Tong, J. Tang, and T. Wang, "Fuzzy adaptive control of multivariable nonlinear systems," Fuzzy Sets and Systems, vol. 111, no. 2, pp. 153-167, 2000.

[15] W. Ma, D. Geng, and Y. Yan, "Multi-phase fuzzy control of single intersection in traffic system based on genetic algorithm," International Journal of Innovative Computing Information and Control, vol. 8, no. 5, pp. 3387-3397, 2012.

[16] S. Tong, T. Wang, and J. T. Tang, "Fuzzy adaptive output tracking control of nonlinear systems," Fuzzy Sets and Systems, vol. 111, no. 2, pp. 169-182, 2000.

[17] Y. G. Leu, T. T. Lee, and W. Y. Wang, "Observer-based adaptive fuzzy-neural control for unknown nonlinear dynamical systems," IEEE Transactions on Systems, Man, and Cybernetics B, vol. 29, no. 5, pp. 583-591, 1999.

[18] C.-C. Kung and T.-H. Chen, "Observer-based indirect adaptive fuzzy sliding mode control with state variable filters for unknown nonlinear dynamical systems," Fuzzy Sets and Systems, vol. 155, no. 2, pp. 292-308, 2005.

[19] S. Tong, H.-X. Li, and W. Wang, "Observer-based adaptive fuzzy control for SISO nonlinear systems," Fuzzy Sets and Systems, vol. 148, no. 3, pp. 355-376, 2004.

[20] C. H. Wang, H. L. Liu, and T. C. Lin, "Direct adaptive fuzzyneural control with state observer and supervisory controller for unknown nonlinear dynamical systems," IEEE Transactions on Fuzzy Systems, vol. 10, no. 1, pp. 39-49, 2002.

[21] C.-S. Tseng and C.-K. Hwang, "Fuzzy observer-based fuzzy control design for nonlinear systems with persistent bounded disturbances," Fuzzy Sets and Systems, vol. 158, no. 2, pp. 164179, 2007.

[22] J. H. Park and G. T. Park, "Adaptive fuzzy observer with minimal dynamic order for uncertain nonlinear systems," IEE Proceedings Control Theory and Applications, vol. 150, no. 2, pp. 189-197, 2003.
[23] A. Wu and P. K. S. Tam, "Stable fuzzy neural tracking control of a class of unknown nonlinear systems based on fuzzy hierarchy error approach," IEEE Transactions on Fuzzy Systems, vol. 10, no. 6, pp. 779-789, 2002.

[24] H. X. Li and S. Tong, "A hybrid adaptive fuzzy control for a class of nonlinear MIMO systems," IEEE Transactions on Fuzzy Systems, vol. 11, no. 1, pp. 24-34, 2003.

[25] T. Shaocheng, C. Bin, and W. Yongfu, "Fuzzy adaptive output feedback control for MIMO nonlinear systems," Fuzzy Sets and Systems, vol. 156, no. 2, pp. 285-299, 2005.

[26] X. Su, P. Shi, L. Wu, and Y. D. Song, "A novel approach to filter design for T-S fuzzy discrete-time systems with time-varying delay," IEEE Transactions on Fuzzy Systems, vol. 20, no. 6, pp. 1114-1129, 2012.

[27] X. Zhang, C. Zhang, and Z. Cheng, "Asymptotic stabilization via output feedback for nonlinear systems with delayed output," International Journal of Systems Science, vol. 37, no. 9, pp. 599607, 2006.

[28] R. Yang, H. Gao, and P. Shi, "Delay-dependent robust $H_{\infty}$ control for uncertain stochastic time-delay systems," International Journal of Robust and Nonlinear Control, vol. 20, no. 16, pp. 1852-1865, 2010.

[29] Y. S. Moon, P. Park, and W. H. Kwon, "Robust stabilization of uncertain input-delayed systems using reduction method," Automatica, vol. 37, no. 2, pp. 307-312, 2001.

[30] A. Germani, C. Manes, and P. Pepe, "A new approach to state observation of nonlinear systems with delayed output," IEEE Transactions on Automatic Control, vol. 47, no. 1, pp. 96-101, 2002.

[31] D. Yue and J. Lam, "Stabilizing controller design for uncertain systems with time-varying input delay," IEE Proceedings Control Theory and Applications, vol. 151, no. 6, pp. 699-705, 2004. 


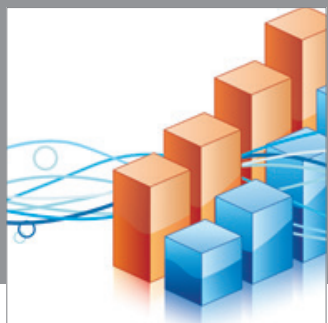

Advances in

Operations Research

mansans

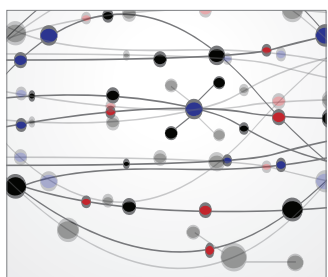

The Scientific World Journal
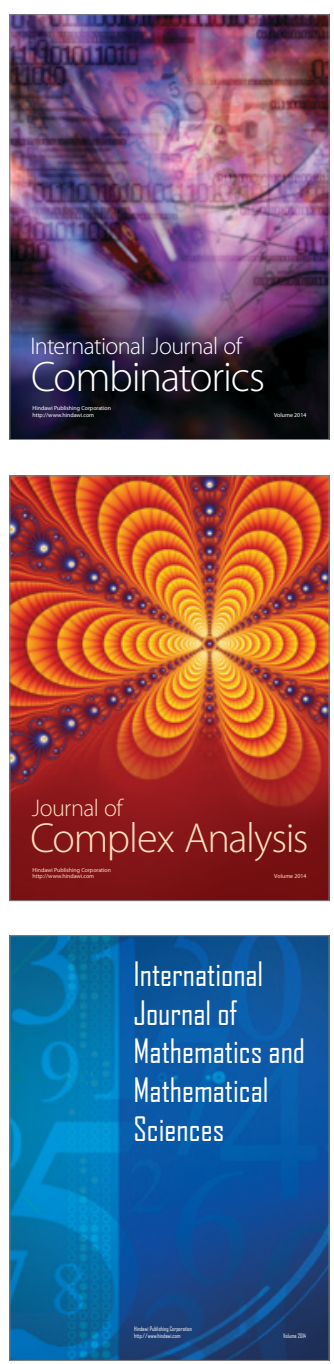
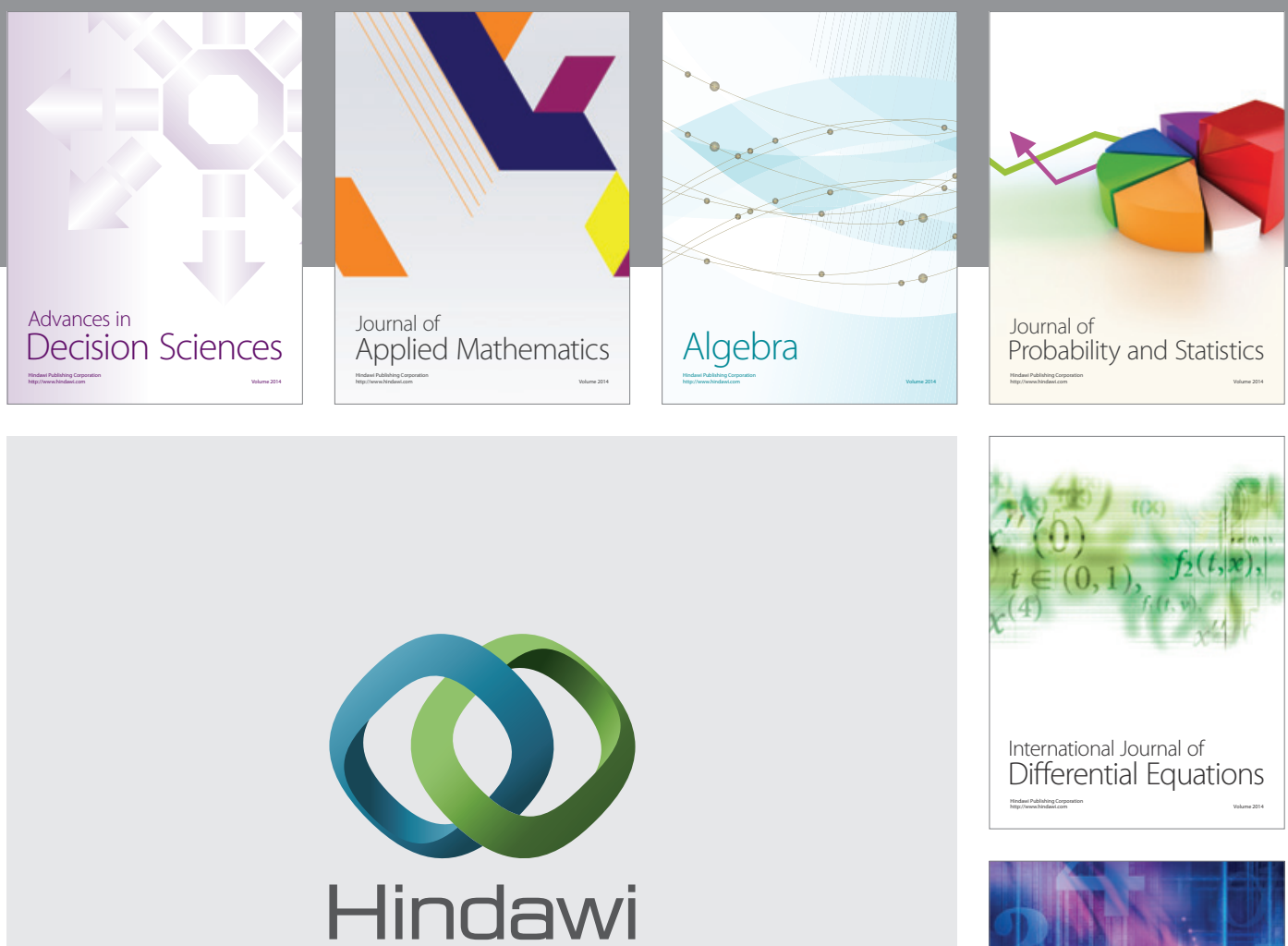

Submit your manuscripts at http://www.hindawi.com
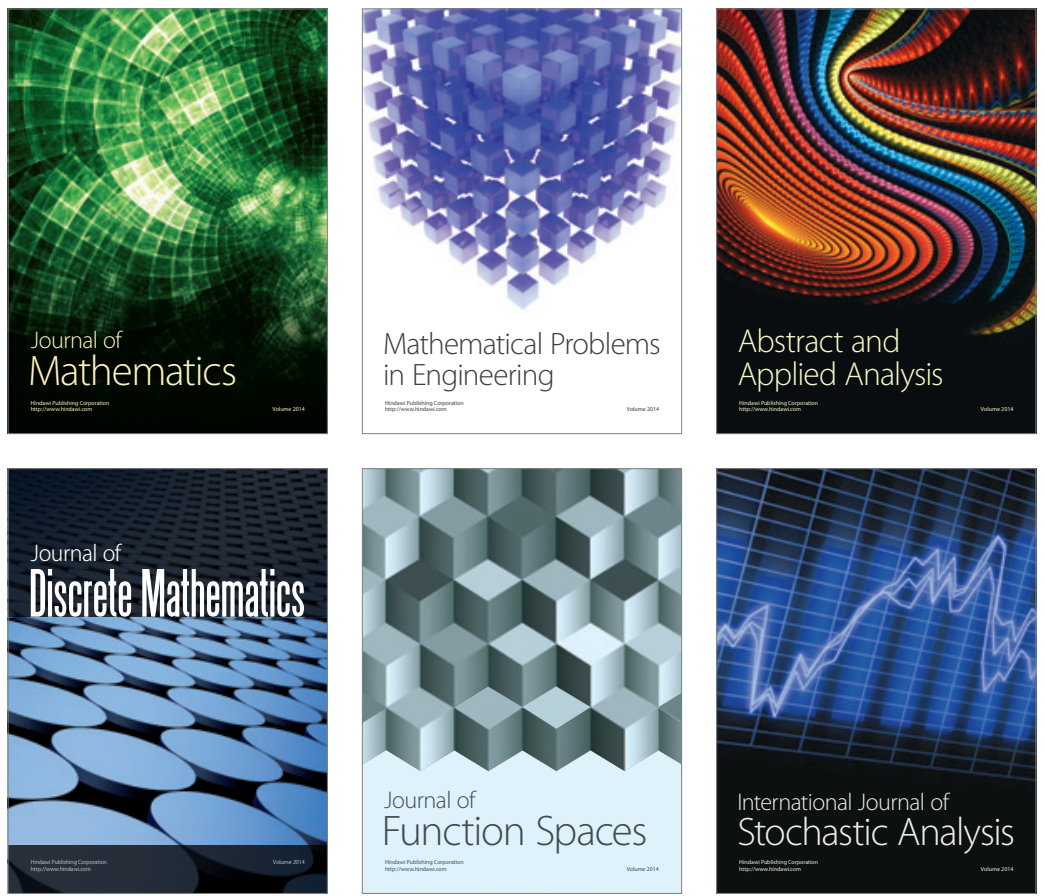

Journal of

Function Spaces

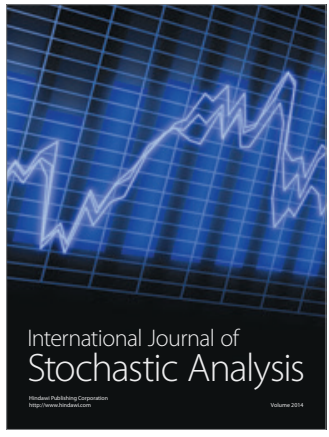

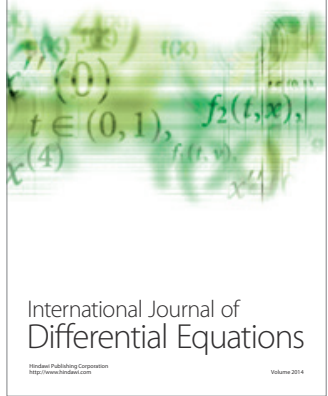
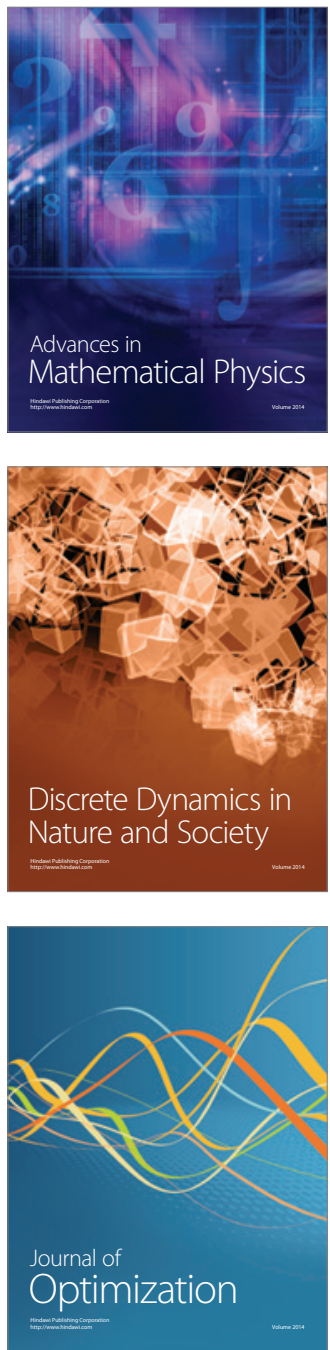